\title{
DAMP: a protocol for contextualising goodness-of-fit statistics in sediment-discharge data-driven modelling
}

\author{
Robert J. Abrahart ${ }^{1}$, Nick J. Mount ${ }^{1}$, Ngahzaifa Ab Ghani ${ }^{1,2}$, Nicholas J. Clifford ${ }^{3}$ and Christian W. \\ Dawson $^{4}$ \\ ${ }^{1}$ School of Geography, University of Nottingham, Nottingham, NG7 2RD, UK \\ ${ }^{2}$ Faculty of Civil Engineering and Earth Resources, Universiti Malaysia Pahang, 26300 Kuantan, \\ Pahang Darul Makmur, Malaysia \\ ${ }^{3}$ Department of Geography, Kings College London, Strand Campus, Strand, London, WC2R 2LS, \\ UK \\ ${ }^{4}$ Department of Computer Science, Loughborough University, Loughborough, LE11 3TU, UK
}

Tel: +44 115846 6145; Fax: +44 115951 5249; Email: bob.abrahart@ nottingham.ac.uk

\begin{abstract}
The decision sequence which guides the selection of a preferred data-driven modelling solution is usually based solely on statistical assessment of fit to a test dataset, and lacks the incorporation of essential contextual knowledge and understanding included in the evaluation of conventional empirical models. This paper demonstrates how hydrological insight and knowledge of data quality issues can be better incorporated into the sediment-discharge data-driven model assessment procedure: by the plotting of datasets and modelled relationships; and from an understanding and appreciation of the hydrological context of the catchment being modelled. DAMP: a four-point protocol for evaluating the hydrological soundness of data-driven single-input single-output sediment rating curve solutions is presented. The approach is adopted and exemplified in an evaluation of seven explicit sediment-discharge models that are used to predict daily suspended sediment concentration values for a small tropical catchment on the island of Puerto Rico. Four neurocomputing counterparts are compared and contrasted against a set of traditional log-log linear sediment rating curve solutions and a simple linear regression model. The statistical assessment procedure provides one indication of the best model, whilst graphical and hydrological interpretation of the depicted datasets and models challenge this overly-simplistic interpretation. Traditional log-log sediment rating curves, in terms of soundness and robustness, are found to deliver a superior overall product - irrespective of their poorer global goodness-of-fit statistics.
\end{abstract}

Key words: data-driven appraisal modelling protocol; data-driven model; neuro-fuzzy model; neural network model; sediment rating curve; suspended sediment concentration; hydrological insight; tropical catchment

\section{INTRODUCTION}

Accurate and reliable suspended sediment estimates are required in a variety of experimental and operational hydrological situations, for scientific and/or river management purposes. Sediment ratings may, for example, be used to estimate long-term rates of landscape denudation; to reflect river morphological changes; to gauge sensitivity of catchments to varying land use practices; or for project specific applications, such as the estimation of reservoir lifetimes, or in identification of tolerable effluent discharge, and/or water quality inputs, around hydroelectric turbines. Accuracy and reliability of such approaches are fundamentally limited: (i) by the quality and quantity of observations (both of which, in turn, reflect sampling design and instrumentation); and (ii) by our ability (or otherwise) to generalise site-specific fluxes to: larger catchment areas; contributing areas of catchments (which are known to be highly variable); and/or event-specific and longer-term flow contributions (where various hysteresis effects are frequently present). This may result in the use of multiple rating curves to model different components of seasonal and hysteresis patterns, or in cases of highly complex responses, it may necessitate the use of process-based models to adequately model the rating relationship. 
Conventionally, rating curves are generated from best fit regressions of suspended sediment - either load (SSL) or concentration (SSC) — against river discharge (Q) or stage (H). Time-varying behaviour may be captured by fitting two curves (where, for example, there is distinct seasonality in sediment supply from a catchment), but where multi-scale temporal and spatial dependency is present or required in the estimation, and/or when physical realism in the link between prediction and predictor is required, then sediment concentration may be modelled as an output from one or more inputs, distributed in time, space, or both. The data-driven model (DDM) offers an important modelling paradigm in such respects, due to its central focus on identifying the computational combination of multiple inputs according to the numerical structures found within a training dataset, and the subsequent re-application of these captured structures to allow for prediction of incomplete data series. Indeed, numerous examples of DDMs in hydrology that focus on suspended sediment prediction have been reported over the last decade. These include individual or modular feedforward neural network prediction (Cigizoglu, 2004; Jain, 2001; 2008); generalized regression neural network prediction (Kisi, 2004a; Cigizoglu and Alp, 2006); radial basis function neural network prediction (Kisi, 2004a; Alp and Cigizoglu, 2007); fuzzy-differential-evolution prediction (Kisi, 2004b: 2009); neuro-fuzzy and fuzzy logic prediction (Kisi, 2005; Kisi et al., 2006); support vector machine prediction (Cimen, 2008); genetic programming prediction (Aytek and Kisi, 2008); and neuro-wavelet and neuro-fuzzy-wavelet conjunction model prediction (Partal and Cigizoglu, 2008; Rajaee et al., 2010).

The number of published papers on suspended sediment prediction in rivers using DDMs is increasing. However, long-standing criticisms that centre on the difficulties associated with generating a physical interpretation of solutions that are commonly presented as an implicit black-box model remain pertinent e.g. Minns and Hall (1996, p.400); Babovic (2005, p.1515); Abrahart et al. (2010). Indeed, the ability of DDMs "to find connections between the system state variables (input, internal and output variables) without explicit knowledge of the physical behaviour of the system" (Solomatine et al., 2008; p.17) can lead to erroneous model conceptualisation and structure (Abrahart et al. 2008) and issues of model equifinality (Beven, 2006; Todini, 2007). Moreover, model choice is commonly justified on the basis of little more than qualitative appraisal of time series graphs, or scatterplots of observed versus predicted records, plus a handful of global goodness-of-fit statistics. This results in models that have little heuristic value beyond that of optimised curve fitting (Mount and Abrahart, in press). Perhaps more dangerously, it may also result in the rejection of more complex and/or more realistic solutions, in favour of simpler, unrealistic counterparts simply on the basis of improved fit statistics (c.f. Oreskes, 2003). Two fundamental concerns therefore emerge:

1. There is an inherent risk in failing to properly understand or appreciate the complexities of a particular dataset. Knowledge of the data quality, the likelihood of significant errors at particular discharge ranges and the extent to which a solution may be influenced by outliers in the dataset is vital.

2. There is a need for meaningful graphical inspections to be performed that assess the appropriateness of each proposed and/or developed solution at different data ranges and with respect to its hydrologic context.

Given these concerns, it is illuminating to contrast the decision making sequence commonly adopted by sediment-discharge data-driven modellers with those engaged in more conventional, empirical modelling approaches and, for whom, the need to provide both hydrological and physiographical context for their results has long been recognised e.g. following the well-established protocol of Glysson (1987). Figure 1 shows the two sequences. The DDM decision sequence is highly simplistic and reliant on statistical fit information to guide the selection of the preferred model. Indeed, it clearly exemplifies the key assumption underpinning data-driven approaches: that the modelling mechanism will learn the required knowledge directly from the data and, in so doing, deliver a preferred model without the need for a priori understanding of either data or hydrological context. By contrast, conventional empirical model decision sequences use contextual understanding to guide the constraints that should be incorporated into a model (i.e. linear, power function) and evaluate model 
outputs in terms of both form and fit to the data. In so doing, a more meaningful feedback loop is incorporated, through which valuable diagnostic information about the model form and fit is used as an additional piece of evidence to: (i) deliver an enhanced understanding of the scientific challenges involved and; (ii) assist thereafter in helping hydrological modeller's to refine their products.

One approach to addressing the above concerns is through improvement of the DDM decision making sequence so that contextual hydrological and data-quality-related understanding is more fully incorporated, with the result that the DDM and conventional empirical decision sequences are more closely mapped. However, this raises the crucial question of how to include and use contextual information to improve model evaluation, whilst adhering to the notion that the data-driven modelling mechanism should be allowed to generate models directly from the data, in the absence of additional knowledge inputs (i.e. external contextual material should not be allowed to act as an a priori guide that is acquired from the modeller). A simple solution is presented in Figure 2. Parallel knowledge about model form and fit, and an understanding of the modelling problem and the hydrological dataset, are conflated and used to guide the preferred model selection. Crucially, contextual understanding of the data is included in the post-modelling phase of the decision sequence; thereby enabling it to be used in the model selection process whilst ensuring that it does not form a preconceived initial input into the data-driven modelling mechanism.

The decision sequence in Figure 2 can be recast to a four-point data-driven appraisal modelling protocol (DAMP). This protocol mimics the classic empirical approach of Glysson (1987) and asserts that the development of DDM solutions for modelling sediment-discharge relations requires the following actions:

1. At the outset, the data are assessed in terms of their physiographic and hydrologic context so that clear hypotheses about the spatial and temporal processes that are expected to be driving suspended sediment over the period of the dataset are developed;

2. The data are examined and reported in depth. The purpose of this analysis is to fully disclose its quality and evidential errors;

3. A synthesis of the main hydrological processes driving the sediment / discharge relationship in the catchment is used to inform an explanation of the resultant structures that one can observe in a standard log-log plot of the paired dataset;

4. The data are next modelled and, if permitted, explicit formulae thereafter developed to represent each model. Each equation is subsequently used to develop and present a regularised data series, from which the performance of each individual solution is examined within its hydrological context and across its data ranges. Finally, the difference in each model's ability to predict suspended sediment in these ranges is identified, and the best performing model is selected on the basis of both goodness-of-fit statistics and the extent to which the model form reflects the catchment's hydrological context and avoids being overly influenced by data quality issues.

Points 1-3 should be easily achieved in all data-driven modelling scenarios. Point 4 raises a significant challenge as many data-driven modelling mechanisms are presumed to be of an 'implicit nature'. However, numerous DDM solutions can be made explicit, by adjusting software settings, or by means of some minor re-coding, such that their internal structures and parameters can be extracted/exported and reported/shared. For example, Lee et al. (2006) provided a neural network (NN) equation for estimating reservoir sedimentation due to typhoon events in Taiwan; Aytek et al. (2008) provided a $\mathrm{NN}$ equation for estimating daily reference crop evapotranspiration in California, USA - albeit that the correctness of the latter has been placed in doubt (Abrahart et al., 2009). The alternative but less attractive option in such cases is to simply run a regular series through each original computer model and publish that paired input-output sequence in a spreadsheet. For those DDMs which do offer explicit outputs, the opportunity to assess the appropriateness of the solution in the context of DAMP is greatly enhanced. For example, in the case of neuro-fuzzy (NF) modelling, it is claimed that "the additional benefit of being able to provide the set of rules on which the model is based ... gives further insight into the process being modeled" (Sayed et al., 2003; p.123: reproduced in the closing 
paragraphs of Kisi, 2005; Kisi et al., 2008, 2009). Consequently, the appropriateness of a NF model's computational structures and rules, may be reasonably assessed by means of detailed referencing and comparison against two independent sources: (i) the structures that can be visualised in the data; and (ii) our domain knowledge of the physical or operational and mechanical processes that are responsible for such structures.

For sediment-discharge rating curves, saved in proprietary model format, or converted into explicit equations it is possible to apply DAMP in full by throughputting an ordered, regular series of inputs, spanning the range of the input predictor values required, and plotting model outputs as an organised sequence of points. Whilst undertaking analyses of this type is unusual, it is not new and a small number of under-appreciated exemplars already exist e.g. the two fuzzy modelling rainfall-runoff relationships plotted in Şen and Altunkaynak (2003; p.42). Indeed, for simple DDMs where the number of predictor variables is low (most sediment-discharge models are of this type), 2D modelled relationships can be depicted using bi-variate plots and 3D modelled relationships can be mapped as surfaces.

In this paper, just such an approach is adopted and we exemplify DAMP by means of a re-evaluation of seven explicit models used to predict SSC from Q for a small tropical catchment in Puerto Rico. The purpose here is not to offer direct insights into better model development strategies, but to highlight the dangers associated with data-driven model selection made on the basis of summary goodness-of-fit statistics alone. Consequently, this paper provides an important foundation/ blueprint for those wishing to present sediment-discharge DDM models in such a way that are better able to respond to the 'black-box demonization' that has plagued them, to date, and in so doing, integrate contextual hydrological and data-related information into model selection and evaluation. The clear weaknesses that have surrounded the use of goodness-of-fit statistics to assess model performance are mitigated and a case for the greater acceptability of DDMs in hydrological modelling is presented. In addition, calls for increased accessibility and portability of hydrological applications (Buytaert et al., 2008; Abrahart et al., 2009) are heeded and the explicit equation for each model in this paper is reproduced and encoded in a spreadsheet for third parties to use in experimental operations (Appendix 1: Supplementary Material).

\section{STUDY SITE AND DATASETS: PHYSIOGRAPHIC, HYDROLOGIC AND SEDIMENTOLOGIC CONTEXT}

\subsection{The study site}

The modelling scenario is one of the two independent case studies that were investigated in Kisi (2005): estimation of United States Geological Survey (USGS) SSC records for upper reaches of the 'Rio Valenciano near Juncos', situated on the island of Puerto Rico, in the Caribbean (USGS Station No. 50056400: Figure 3). The sediment budget at this gauging station is of particular hydrological modelling interest since the Commonwealth of Puerto Rico, Aqueduct and Sewer Authority are constructing a $30 \mathrm{~m}$ high dam on this river to meet increased water demand: situated about $2 \mathrm{~km}$ south of the city of Juncos (http://www.csagroup.com/project.php?msid=1\&pid=40).

The USGS monitoring site is located at an elevation of approximately $70 \mathrm{~m}$, roughly halfway between the river's source in the hills above Las Piedras, and the confluence of the Rio Valenciano and Rio Grande de Loiza in the alluvial plains of the Cagus-Gurabo-Juncos region. The catchment area above the gauge is $42.4 \mathrm{~km}^{2}$ and rises to approximately $400 \mathrm{~m}$ at its highest point. The river flows through mainly suburban land use for about $7 \mathrm{~km}$ immediately upstream of the gauge (Pares-Ramos et al., 2008) where a relatively low relief landscape is characterised by a mix of grassland and low density urban development. This land use changes to a rural classification in the uppermost sub-catchments. In common with the majority of upland rivers on this island, the rural upland reaches of the Rio Valenciano are highly incised and bedrock controlled. Floodplains are generally poorly developed and the channel is commonly highly connected to the valley sides; resulting in few opportunities for outof-channel sediment storage. Forestry remains the primary land use type on the steepest valley sides and in the highest elevation first order sub-catchments. Much of the upland catchment has however 
been deforested: a process resulting in the replacement of natural forests by grassland, shrub land, arable agricultural land or plantations (Helmer et al., 2002; Pares-Ramos et al., 2008). Elevated ridges in the upper reaches are characterised by low density urban development.

This catchment is one the many northward draining examples on the island that are characterised by high mean annual rainfall of between 2000 and $2500 \mathrm{~mm} \mathrm{yr}^{-1}$ (Larsen and Torres-Sanchez, 1998), moderate to high annual sediment yields and moderate to high annual runoff (Warne et al., 2005). Rainfall is temporally variable with low-intensity localised rainfall events tending to be fairly evenly distributed throughout the driest months of the year (January to April); resulting in a persistent lowlevel base flow in the majority of northern catchment channels. By contrast, mean rainfall doubles during the wetter months of May to October and, in some larger catchments, such rainfall can result in sustained moderate discharges. The island also experiences occasional, extreme rainfall-runoff events associated with hurricanes that recur over 10-20 year periods (Ho, 1975; Nueman et al., 1990; Scatena and Larsen, 1991). Indeed, although infrequent, hurricane-related events are important for generating sediment, with 285 shallow landslips reported by Scatena and Larsen (1991) in upland forested valleys during the passage of Hurricane Hugo (1989). The upper Rio Valenciano catchment for example was classified as having moderate landslide susceptibility by Larsen and Torres-Sanchez (1998). The remaining winter months are dominated by localised high intensity rainfall events linked to annually-recurring winter frontal storms. Major storms are intense but brief and in response most catchments on the island exhibit very flashy hydrological regimes in which maximum discharges can be up to four orders of magnitude above base flow, yet recede over hours, or at most a few days (Warne et al., 2005). In consequence, the occurrence of high sediment yields in northern catchments is often episodic with the highest sediment concentrations related to short-lived flood events associated with storms and landslides.

There are no detailed geomorphological and physiographic studies of the catchment available in the literature, although studies in the nearby Luquillo Mountains (USGS, 2000) provide the best contextual data available for its upland reaches. It is, therefore, not possible to a priori suggest either: (i) a definitive sediment budget; or (ii) specific elements of a sediment yield/delivery system (Dunne, 1979). Indeed, in a study of small basins in Puerto Rico, Larsen (1997) drew attention to marked local variability in basin response, which reflected catchment physiography; underlying geology; history of land use, and/or past and present land use clearance; and adjustments of the stream network with respect to local sediment storages in bar forms, and to channel-hillslope interactions. It follows from this, that any dataset must be closely tied both to the particular basin under consideration and to the position of the measuring station in relation to the stream network - since the influence of very local network sediment storages and supplies may be evident in any data records. However, comprehensive studies of other northern catchments on the island (e.g. Larsen, 1997; Larsen and Torres-Sanchez, 1998; Warne et al., 2005; Diaz-Ramirez et al., 2008) do allow the general characteristics of sediment dynamics to be posited. These indicate that a high degree of seasonality in sediment erosion, and hence of sediment supply to the stream network, is to be expected in conjunction with a rapid transit of suspended sediment through the upland stream network. The effect may be complicated (probably compounded) since the 'hurricane season' also corresponds with the wettest months i.e. the largest, most intense precipitation events, coincide with an already wet period. Event-specific highs in sediment yield associated with landslides and localised soil erosion - if present - could thus be superimposed on higher, seasonal values. The presence of 'extreme' values, is, therefore, to be expected, and such occurrences may well have a disproportionately large influence on sediment transport and suspended sediment concentration in relation to their frequency of occurrence. However, given the small size of most catchments on this island, it may also be the case that seasonal effects are further complicated by local variations in sediment availability, if not delivery: large rainfall events early in the wetter season might, therefore, be expected to produce a higher sediment yield than events occurring later in the season, when more of the available sediments have been eroded. However, this will depend upon local land stability conditions and any changes in land use practice. Some upper-end tailing-off or dipping of rating curves might also, then, be anticipated. The net effect of all of the above might most likely be encountered in the portrayal of a two- or multi-stage log-log regression relationship, between sediment concentration and discharge (principal seasonal 
effect). That relationship might also be expected to exhibit differing levels of scatter about the individual stages: (i) as a result of event-specific factors; (ii) due to extrapolation and infilling of missing records: or (iii) associated with different types of hysteresis, where similar sediment concentrations are recorded for very different discharges (Williams, 1989).

\subsection{The datasets}

Full particulars for the monitoring station at Rio Valenciano near Juncos are provided in Table 1. Following Kisi (2005), paired time series datasets comprising daily river discharge $\left(\mathrm{Q}\right.$ in $\left.\mathrm{m}^{3} \mathrm{~s}^{-1}\right)$ and daily SSC $\left(\mathrm{S}_{\text {in } \mathrm{mg} \mathrm{l}^{-1}}\right.$ ) records for 1 October 1993 - 30 September 1995 were downloaded from the USGS open access website at: http://webserver.cr.usgs.gov/sediment (Figure 4).

Little additional metadata is available from the download site, so additional information related to gauge instrumentation, and any data processing or infilling, was requested directly by the authors (Carlos Figueroa-Alamo, USGS, PR, pers. comm.). This request revealed that SSC records for low to moderate discharges were collected using a manual depth-integrated sampler. For high discharges an automatic sediment sampler with liquid level actuator (non-depth integrated) was used. Sampling frequencies are not disclosed. Importantly, it is also revealed that field-based SSC sampling was not continuous and that data infilling was necessary for some SSC records; particularly those at low discharges. The methods by which this infilling was accomplished are not detailed. Similarly, for the highest discharges SSC is extrapolated via rating curves; but the form and reliability of these curves remains unclear.

The raw dataset is presented as a log-log plot in Figure 5. The observed sediment-discharge relationship, could obviously be reasonably-well captured by means of log-log regression, with varying degrees of scatter due to seasonal and/or event-specific factors, and exhibiting a general reduction in the gradient of the curve in the uppermost ranges of the dataset (limited sediment availability). However, a number of important additional structures can also be observed. The degree of scatter associated with the four extreme discharge events (>15 cumecs) is relatively low and the expected high degree of heteroscedasticity in the dataset may be artificially constrained by the application of upper-magnitude extrapolation procedures. The scatter of points below 0.5 cumecs is characterised by numerous horizontal 'lines of points' (A); implying that SSC is constrained to only one of seven or eight possible values. Each particular value also occurs across a range of overlapping discharges. Clearly, such records are not realistic. This regular pattern is most likely an artefact arising from measurement and recording imprecision at very low levels of SSC i.e. discretisation/ round-off. It could even, perhaps, be an unintended by-product of missing record "infilling operations". This particular activity was mentioned in the personal communication and might help in part to explain the extent of their horizontal spread. Contrasting vertical 'lines of points' (B) also exist in the data between 0.5 and 0.8 cumecs; suggesting that wide ranges of SSC values have been recorded for identical discharges. Again, this is highly unlikely, and one may reasonably presume that these structures are due to a reduced precision in the discharge record that has resulted in SSC data being assigned to one of a small number of data 'bins'. Two significant outliers also exist (O1 and O2) whose validity it is difficult to imagine: 24 April 1994 (Flow $=0.0906 \mathrm{~m}^{3} \mathrm{~s}^{-1}$; Sediment $=1200$ $\mathrm{mg} \mathrm{l}^{-1}$ ) and 6 June 1994 (Flow $=0.2270 \mathrm{~m}^{3} \mathrm{~s}^{-1}$; Sediment $=312 \mathrm{mg} \mathrm{l}^{-1}$ ). Indeed, the most likely explanation for these peculiarities is data error in the source material.

This initial appraisal of the dataset, coupled with the physiographic, hydrologic and sedimentologic context, raises some important issues for those wishing to generate a DDM of suspended sediment for the Rio Valenciano. First, the data frequency (daily mean) does not correspond with the expected hydrological event frequency (commonly less than 24 hours); potentially resulting in a significant underestimation of the instantaneous 'peak value' for SSC in cases of a rapid and/or larger event. Second, using data extrapolation procedures for high discharges may be problematic, since it results in records that do not adequately capture the variability of the sediment supply and transport processes that occur within a catchment under extreme conditions. Consequently, the occurrence of extreme events in the dataset may be constrained. Given the fact that majority of suspended sediment yield in northern catchments is associated with flashy, high discharge events, this also raises questions over 
the usefulness of such time series records for modelling SSC. Moreover, the relatively simple form of most rating curves, can easily be replicated by most DDMs, such that the vast majority of resultant models will appear to possess high levels of skill if assessed in terms of goodness-of-fit statistics; thereby giving a false indication of the validity of the model at high discharges. Third, clear shortcomings exist with regard to measurement and recording precision of SSC. This has resulted in a record that fails to properly represent a tight sediment-discharge relationship at low discharges. The combined depiction of inappropriate scatter and artificial structures moreover delivers a substantial amount of uncertainty as to what does or does not constitute a correct answer in that region. The extent to which such particular imperfections could have a detrimental impact on a potential solution is nevertheless open to question: it may have little operational relevance for the resultant model, since the contribution to overall suspended sediment loads from low discharges is likely to be minimal. However, given the higher frequency of low discharge records in each dataset, a large proportion of the data used to train a DDM may not be representative of real suspended sediment behaviour, such that the ability of the model to produce realistic predictions at low discharges may be impaired. Finally, the existence of the two outliers in the data are likely to encourage a general over-estimation of SSC at discharges < 1 cumec.

\section{METHODOLOGICAL APPROACH}

The underlying methodology is that of Kisi (2005); extended as required to support a more comprehensive analysis by means of DAMP:

1. Download dataset and divide it into two subsets: (Set A) and (Set B);

2. Develop a number of DDMs on Set A and test on Set B (Experiment 1);

3. Develop a number of DDMs on Set B and test on Set A (Experiment 2);

4. Extract each model's rules, weights, parameters and governing equations (as required);

5. Use these rules to develop and present a regular data series such that the modelled relationship between SSC and Q can be plotted as a continuous curve;

6. Compare goodness-of-fit statistical performances for all models using the two contrasting datasets;

7. Examine how well each regular curve performs with respect to its hydrological context in particular ranges of the original dataset; as outlined above.

The downloaded material was first divided into two equal and consecutive subsets. Set A (Kisi's training dataset) contained 365 paired daily values for the 1994 water year (1 October 1993 - 30 September 1994); Set B (Kisi's testing dataset) contained 365 paired daily values for the 1995 water year (1 October 1994 - 30 September 1995). Earlier studies, however, had included a lagged input of 1-day: meaning that no prediction could be delivered for 1 October. This necessitated removal of the observed record for 1 October 1993 from Set A and 1 October 1994 from Set B: leaving 364 records in each set. Table 2 provides a statistical description of each variable in each final subset and a number of single measurement inequalities can be identified. The highly skewed and highly peaked nature of discharge and sediment in both catchments should be emphasised. Table 2 also reveals potentially problematic differences in the split of observed records that could create a substantial impact on overall outcomes. The split-distribution is not well balanced: the calibration and testing datasets possess marked disparities in terms of mean, skewness and kurtosis. The extent to which relational or covariant inequalities might be an issue is not revealed in such descriptors. Last, but by no means least, no error checking, outlier removal or data cleansing operations were reported in the original paper and so the assumption must be that none of the original downloaded records should be deleted. Consequently, the two erroneous outliers remained.

To allow direct comparison with Kisi's earlier paper the four models that he reported are replicated here. These are a neuro-fuzzy model possessing triangular membership functions (NFT); a neural network model possessing a linear transfer function in its output unit (NNL); a traditional power function sediment rating curve model (PFT) and a simple linear regression model included for the purposes of 'linear benchmarking' (SLR). In addition we also present results from a neuro-fuzzy 
model possessing Gaussian membership functions (NFG); a neural network model possessing a nonlinear transfer function in its output unit (NNN) and a bias-adjusted power function sediment rating curve model (PFA). Therefore, a total of seven separate modelling approaches are presented.

In accordance with the approach adopted in Kisi (2005), each of the seven independent models was calibrated on data Set A and tested on Set B, identified by means of a prefix attached to each model, in this case 'MOD'-1 This scenario allowed direct comparison between the numerical assessment statistics presented in the earlier paper and the establishment of some measure of correspondence, or fidelity, in terms of replication with regard to the recreation of earlier reported solutions. However, in addition, we also adopted a 'role reversal test' by comparing and contrasting models calibrated on Set B and tested on Set A ('MOD'-2). The object of this particular exercise was to test for a consistent response, since from earlier discussions, it was suspected that disparities related to the original selection of appropriate subsets for calibration and testing purposes could be influential and had perhaps led to a unique set of findings. Consequently, 28 sets of numerical predictions are reported for subsequent out-of-sample assessment in terms of conventional statistical methods.

Three methods of reporting are adopted. Each model comprises an explicit set of mathematical rules and/or equations that can be used to describe the form of the relationship and in so doing will provide an exact method, for comparing and contrasting the different types of solution, which is also transparent. The equations are provided below and in the accompanying spreadsheet (Appendix 1: Supplementary Material). Having both computational and mathematical models at our disposal means that it is possible to obtain outputs for a regular series of pre-specified discharge inputs, in this case ranging from some practical minimum [0] to some upper operational limit [50] that exceeds the maximum observed record [35.1] for Q. The resultant outputs can thereafter be plotted as a continuous function for visualisation purposes in the same manner as a simple linear regression equation overlaid on the original scatterplot of observed S vs. observed Q. The extent to which each model fits the dataset can thereafter be inspected for hydrological correctness. Particular emphasis should be placed upon: (i) the development of a flexible nonlinear response, accommodating local deviations from the global trend; and (ii) the treatment of outliers. The use of an extended series that is expanded well beyond the upper range of the observed dataset is particularly useful in exploring the extrapolation capabilities of each model that was produced. Individual model outputs were also compared and contrasted against one another by means of seven popular metrics computed using HydroTest (www.hydrotest.org.uk): an open access web site that performs the required numerical calculations in a standardised manner (Dawson et al. 2007; 2010). The selected statistics comprised: three absolute measures - Mean Absolute Error (MAE), Mean Error (ME) and Root Mean Squared Error (RMSE); three relative measures - Mean Absolute Relative Error (MARE), Mean Relative Error (MRE) and Mean Squared Relative Error (MSRE); and one dimensionless coefficient - RSquared (RSqr). Full details with regard to the calculation and use of such descriptors, are provided in the aforementioned papers. Table 3 contains formulae for and descriptions of all seven metrics: noting that signed statistics are reported as a residual, not an error, such that a positive residual equates to a negative error. The total annual sediment flux for each model and period is also of interest and was calculated separately in metric tons per water year. It is reported in percentage format as Total Error (TE): a measure that is signed according to error, not residual. In practical terms TE provides a simple, but nevertheless very useful, additional statistic: one that places added weight on higher flows/concentrations. This particular weighting is especially important, given that events of this nature will make a significant contribution in terms of overall yield, and that issues surrounding the latter are what many, if not most, practitioners are mainly concerned about.

\section{MODELLING OPERATIONS}




\subsection{Neuro-Fuzzy Models}

Neuro-fuzzy modelling was performed in MATLAB using the Adaptive Neuro-Fuzzy Inference System (ANFIS: Jang, 1993; Jang et al., 1997). Kisi (2005) employed triangular membership functions in all reported applications. That original analysis is extended in this paper to encompass an assessment of two different internal membership functions: triangular and Gaussian. NF models containing either one or other type of internal membership function were developed to meet the demands of each individual experiment. Each NF model involved was designed to be consistent with the best performing NF solution of Kisi (2005). No pre- or post-processing operations were applied. Each model used one raw input, to deliver one raw output, and contained two internal membership functions - as depicted in Figure 6. This is the simplest possible model that the software package will support. It also means that only two rules and two pertinent, appropriately weighted linear output equations will be produced; delivering one internal rule for each of the two individual input membership functions involved. Kisi (2005) did not report the training period or stopping condition that was used to develop his NF model; our assumption, under such circumstances, is that his models were trained for 10 iterations - this number comprising a default setting in the MATLAB Fuzzy Logic Toolbox. Testing was nevertheless performed to ascertain the correctness of our model fitting activities, since it is possible that the resultant solutions could be either underfitted or overfitted. Figure 7 depicts regular series outputs for NF models developed using a limited set of different iteration settings: starting at 5 (half of default), and thereafter doubling to 10 (default), 20 and 40. NFT-1 and NFT-2 outputs revealed a progressive process of continued adjustment, such that additional iterations delivered a series of substantial modifications causing each NFT model to provide very different high-end output trajectories at different stages of the training process. The need to perform some sort of 'early stopping' operation is thus indicated, perhaps founded on the prudent use of a cross-validation dataset. However, such considerations and procedures are not part of the original published methodology, and so regardless of other factors the default number of iterations was applied in our final modelling exercises. NFG-1 and NFG-2 outputs in contrast depicted limited overall changes and a more 'stable solution': such that the use of default settings could not as a result be faulted.

For each finished product, the software package provided modelling parameters for two fitted membership functions, enabling the relevant internal weights to be calculated. It also produced a set of rules and for each rule it supplied a linear output equation. Full particulars are listed in Table 4. The two rules are quite simple and are combined in the region of 'membership function overlap' - as depicted in Figure 8. Thus:

Rule 1: If $\mathrm{Q}$ is $\mathrm{MF}_{1}$ then $\mathrm{S}$ is $\mathrm{S}_{1}$

Rule 2: If $\mathrm{Q}$ is $\mathrm{MF}_{2}$ then $\mathrm{S}$ is $\mathrm{S}_{2}$

Rule 3: If $Q$ is $M_{1}$ and $M_{2}$ then $S$ is $S_{1}+S_{2}$

It is also possible to demonstrate in an exact manner how our modelling outputs can be computed using the information that is provided in Table 4. The calculation of model output (S) can be simplified into a three-step procedure:

1) Calculation of weights. The membership score (weight) for each input is obtained using the parameters listed in Table 4. For NFT the triangular curve is a function of vector Q, and depends on three scalar parameters $a$ (left foot), $b$ (peak), and $c$ (right foot). From these parameters, the weight of each rule (Wi) is calculated as:

If $\mathrm{Q} \leq \mathrm{a}$ then $\mathrm{Wi}=0$

If $\mathrm{a} \leq \mathrm{Q} \leq \mathrm{b}$ then $\mathrm{Wi}=\frac{x-a}{b-a}$ 
If $\mathrm{b} \leq \mathrm{Q} \leq \mathrm{c}$ then $\mathrm{Wi}=\frac{c-x}{c-b}$

If $\mathrm{Q} \geq \mathrm{c}$ then $\mathrm{Wi}=0$

whilst for NFG, the weight of each rule (Wi) is calculated as:

$\mathrm{Wi}=e^{\frac{-(x-c)^{2}}{2 \sigma^{2}}}$

The weight for each rule is thereafter normalised i.e. divided by the 'sum of weights'. For example, the normalised weight $\overline{W_{i}}$ for Rule $\dot{t}$ from $n$ number of rules is calculated as:

$\overline{W_{i}}=\frac{W_{i}}{W_{i}+W_{i+1}+W_{i+2}+. .+W_{\mathrm{n}}}$

2) Calculation of sub-outputs. The linear function related to each particular rule is next multiplied by its corresponding normalised weight e.g., $\overline{W_{1}} \cdot S_{1}, \overline{W_{2}} \cdot S_{2} \ldots \overline{W_{\mathrm{n}}} \cdot S_{\mathrm{n}}$. This step weights the strength of each rule and provides sub-output to the final output.

3) Calculation of final output. The sub-outputs are thereafter summed to provide the final output:

$S=\sum_{i=1}^{n} \bar{W}_{i} \cdot S_{i}$

For example, $\mathrm{S}$ at $\mathrm{Q}=0.425$ in NFT-1 is calculated as follows:

$S=\overline{W_{1}} \cdot(-226.4 Q+4.912)+\overline{W_{2}} \cdot(-270.7 Q+10560)$

where

$\overline{w_{1}}=\frac{w_{1}}{w_{1}+w_{2}} \quad ; \quad \overline{w_{2}}=\frac{w_{2}}{w_{1}+w_{2}} \quad ;$

$w_{1}=\frac{c_{M F_{1}}-Q}{c_{M P_{1}}-b_{M{ }_{1}}} \quad ; \quad w_{2}=\frac{Q-a_{M F_{2}}}{b_{M F_{2}}-a_{M{ }_{2}}} ;$

and where $a, b$ and $c$ are values listed in Table 4 .

\subsection{Neural Network Models}

Neural network modelling was performed using an in-house software program that has delivered sound performance on several previous occasions e.g. Dawson et al. (2002; 2006). Kisi (2005) employed a linear output transfer function in all reported applications. This is the default setting in MATLAB. That original analysis is extended in this paper to encompass an assessment of two different output transfer functions: linear and nonlinear. NN models containing either one or other type of output transfer function were thus developed to meet the demands of each individual experiment. Each NN model involved was designed to be consistent with the best performing NN 
solution of Kisi (2005). Each model used one raw input, to deliver one raw output, and contained one hidden unit - as depicted in Figure 9. Each NN model was trained using 'back propagation with momentum' for 10,000 epochs: for a detailed account of relevant neurocomputing terms and procedures see Priddy and Keller (2005). This is the simplest hidden-unit architecture permitted. Kisi (2005) did not report his training parameter settings or, indeed, if some method of standardisation should be applied to either the input or output datasets. Thus we opted to use a tried and tested approach, that had been used successfully on a number of previous occasions, comprising: a training rate of 0.1 , a momentum setting of 0.9 , and datasets standardised to range from 0.1 to 0.9 . To support operational considerations, each model run was performed in a blind manner, requiring both training and testing datasets to be standardised to the range of the training dataset.

The two NNL and two NNN models can be explained, using a sequence of equations. Figure 9 depicts the $\mathrm{NN}$ architecture that was adopted, comprising one unit in the input layer (i), one unit containing a 'sigmoid activation function' in the hidden layer (j), and one unit that could contain either a linear activation function (as shown) or a sigmoid activation function (not shown) in the output layer (j). No processing occurs in the input layer. Inputs are simply passed to the hidden layer in which the processing operation delivers outputs $\left(\mathrm{S}_{\mathrm{h}}\right)$ using a sigmoid transformation in accordance with Equation 9.

$S_{h}=\frac{1}{1+e^{-\left(Q_{s t d^{\circ}} W 1+B 1\right)}}$

where $Q_{\text {std }}$ is obtained using Equation 10.

$Q_{\text {std }}=\frac{Q-Q_{\min }}{Q_{\max }-Q_{\min }} \cdot 0.8+1$

$\mathrm{S}_{\mathrm{h}}$ is thereafter passed to the output layer, and processed according to one or other of the two potential transformations, linear or non-linear. Final output for NNL is calculated using Equation 11; final output for NNN is calculated using Equation 12.

$S_{\text {std }}=S_{h} \cdot W 2+B 2$

$S_{s t d}=\frac{1}{1+e^{-\left(S_{h} \cdot W 2+B 2\right)}}$

where $S_{\text {std }}$ is then de-standardised back to the original scale by Equation 13 to get the final output $S$.

$S=\left(S_{s t d}-0.1\right) \cdot \frac{S_{\max }-S_{\min }}{0.8}+S_{\min }$

Trained parameter settings obtained for Equations 9 to 13 are provided in Table 5.

\subsection{Sediment Rating Curves}

Two established statistical solutions were developed in Microsoft Excel: a traditional sediment rating curve (PFT) and a bias-adjusted sediment rating curve (PFA). The sediment rating curve method is attributed to Campbell and Bauder (1940) who observed that the relationship between the logarithm of sediment concentration and the logarithm of discharge is approximately linear. PFT is a straightforward least squares linear regression model of $\log \mathrm{S}$ on $\log \mathrm{Q}$. Equation 14 depicts this relationship in the form of a power function where $\mathrm{a}$ and $\mathrm{b}$ are constants acquired during the 
regression process. However, a sediment rating curve that is developed in this manner, should of course be corrected for bias related to the log transformation process given that the differences in means of logged and non-logged values would lead to underestimations. Ferguson (1986) highlighted such issues and demonstrated the use of a bias correction factor (CF: Equation 15).

$S=a Q^{b}$

$S=C F \cdot a \cdot Q^{b}$

where $\mathrm{CF}=e^{2.65 s^{2}}$ and $\mathrm{s}$ is the standard error of the regression equation

The rating curve is a simple method that is in widespread use, and is considered to be adequate for most practical purposes. It nevertheless remains an empirical result without physical justification. The relevant PFT and PFA equations for both Experiment 1 and Experiment 2 are provided in Equations 16 to 19.

$$
\begin{aligned}
& S_{P F T-1}=62.42 Q^{0.9289} \\
& S_{P F A-1}=C F \cdot 62.42 Q^{0.9289} \text { where } C F=1.13556 \\
& S_{P F T-2}=61.79 Q^{1.0482} \\
& S_{P F A-2}=C F \cdot 61.79 Q^{1.0482} \text { where } C F=1.16360
\end{aligned}
$$

\subsection{Linear Regression Benchmark}

Simple linear regression is a popular method for modelling the form of the relationship that exists between two variables in numerous field of science and engineering. It works by fitting a straight line to the set of points that minimises the sum of squared residuals. That line is also required to pass through the centroid (intersection of means) and on this occasion is used to deliver the regression of $\mathrm{S}$ (predictand) on Q ( predictor), according to Equation 20, in which $a$ and $b$ are the slope and intercept of the linear model that is produced.

$S=a+b Q$

Abrahart and See (2007) argued that for modelling purposes simple linear models should be used as standards against which more powerful solutions are tested so as to indicate the degree to which the observed relationship that needs to be modelled is linear and therefore requires the application of a linear modelling solution, as opposed to something more complex/ challenging. The provision of a simple linear solution in such cases for 'benchmarking purposes' must also be clarified: such solutions are not expected to be winners. Their role is to provide a lowest possible standard, such that the degree to which superior solutions are superior can be judged against it. Thus, for benchmarking purposes, two simple linear models (SLR-1 for Experiment 1 and SLR-2 for Experiment 2) were developed and are presented in Equations 21 and 22.

$$
\begin{aligned}
& S_{S L R-1}=18.929+37.482 Q \\
& S_{S L R-2}=14.237+54.665 Q
\end{aligned}
$$




\section{RESULTS}

\subsection{Model comparison with original study}

Three metrics reported in Kisi (2005) can be used to deliver an objective comparison for the purpose of ascertaining the overall extent to which our one-input one-output NFT-1 and NNL-1 models are analogous to their published equivalents (Table 6). The original models were assessed on Mean Root Squared Error (MRSE), RSqr and TE. MRSE is computed from RMSE according to Equation 23.

RMSE $=$ MRSE $\cdot \sqrt{n}$

where $n$ is the number of records, which is 364 . TE was labelled "relative error" in the previous paper.

The statistical results are quite similar, as expected. The original NFT-1 and NNL-1 models nevertheless exhibit marginal albeit inconsistent overall superiority to their latest replicated counterparts. It is impossible to establish to what extent the observed discrepancies can be attributed to random processing elements such as the use of different architectural initialisations or computational precisions. The underlying similarities are nevertheless sufficient to corroborate the key findings of Kisi (2005). These are:

1. statistics for NFT-1 are slightly better than those for NNL-1,

2. PFT-1 and SLR-1 do not perform as well as the other two models.

\subsection{Statistical metrics}

Table 7 contains a full set of output evaluation statistics for Experiment 1. NN and NF solutions provided somewhat similar measures of global fit, to the extent that there might be no significant or meaningful statistical difference occurring, between each individual model, especially in the training situation. Numerical results, for fitting models to the training dataset, are mixed - with no clear winner emerging. NN models are perhaps superior. NNL-1 had a slight advantage in terms of MAE. NNN-1 had an even narrower advantage in terms of RMSE. RSqr was identical in all four cases. NFT-1 and NFG-1 had zero ME meaning that such models were unbiased. Testing, as might be expected, provided similar measures of statistical fit for NN and NF solutions but with NFT-1 on this occasion doing somewhat better than the others on MAE, ME, RMSE and RSqr. The assumption in such cases, if one is possible, is that lower training performance of NFT-1 on Set A resulted in superior generalisation performance on Set B; whilst the tighter fit that NNL-1 and NNN-1 achieved on training Set A, handicapped the transfer of such modelling solutions, in terms of their fit to Set B. This interpretation of matters, however, is to some extent contradicted by the fact that NNN-1 returned the top score for TE: conceivably making it the practitioner's choice.

Table 8 contains a full set of output evaluation statistics for Experiment 2. The statistical results obtained using role reversal, are somewhat different, to that reported for Experiment 1. Three out of four NN and NF solutions provided similar measures of global fit. NNL-2, surprisingly, performed a great deal worse than the other models. Further investigation revealed that this minimalist model lacked sufficient internal flexibility to accommodate the required solution; although by paralleling the principal trend, it nevertheless secured the highest score for RSqr. NNL-2 is, as a result of such failings, not included in the following detailed comparison and analysis. In terms of training, NF models were best. NFG-2 provided an overall champion in terms of MAE and RMSE. NFT-2 was the second best model. NF models had an identical score for RSqr. NFT-1 and NFG-1 also had zero ME meaning that such models are, once again, unbiased. NNN-2 had a marginally lower RSqr. Testing, as might be expected, provided similar measures of statistical fit for NN and NF solutions but on this occasion, produced a greater mixing of positions. NFT-2 was best at MAE, NNN-2 was best at RMSE, whilst NFT-2 and NNN-2 had identical scores for RSqr. NNN-2 in a similar manner to NNN- 
1 returned the top score for TE: thus reinforcing our earlier statement about practioner preference for NNN.

Tables 7 and 8 also contain a set of output evaluation statistics for our four traditional approaches. These particular mechanisms were included for benchmarking purposes and it must be stressed that such models are not intended to be candidate solutions: but to act as standards, that should be bettered. The neural models are clearly better in terms of absolute statistics that place stronger emphasis on higher magnitude sediment events; although PFT is observed in both experiments to offer the best performing solution according to our relative metrics i.e. MARE, MRE and MSRE. The general meaning in such cases is not that traditional approaches should be pursued but, instead, that relative metrics offer an alternative environmental perspective in regard to providing a proper test for the purposes of identifying good or bad models on a specific dataset, or perhaps in a broader sense, on this particular hydrological modelling topic.

\subsection{Regular data series plots}

Figure 10 (Experiment 1/ Trained on Set A) and Figure 11 (Experiment 2/ Trained on Set B) show that most models follow similar trajectories in the central region of each plot. The upper and lower magnitudes are nevertheless modelled in a number of different manners. The upper region of each plot is of particular interest. Figure 10 shows that NFT-1 and NFG-1 display erratic, sharp declines beyond our maximum $\mathrm{Q}$ in the training dataset, with similar difficulties encountered with NFT-2 (Figure 11). NFG-2, by contrast, displays a sharp increase outside of the data range. Indeed, it appears that NF models are particularly prone to localised overfitting in sparse data ranges and offer poor potential for extrapolation. NNL-1, NNN-1 and NNN-2 are more robust but simply flatten out in the upper region so as to target one or more final point(s). However, poor extrapolation capabilities are to be expected, since such models are given no help at all with regard to the existing underlying form. Yet: (i) upper end data is suspect; (ii) upper end events are responsible for a significantly disproportionate amount of total sediment flux; and (iii) a useful model should be able predict beyond the (limited) range of its training dataset in order to characterise events that are more extreme than ones previously encountered. Thus realistic extrapolation, particularly on the upper end, is essential. NNL-2, moreover, performs very poorly overall: in clear contrast to other NN models. It is, nevertheless, by far the best example of our argument that statistical evaluation based on goodness-offit metrics, particularly in the absence of graphical illustration, can be deceiving. It was quite astonishing to discover that a parsimonious data-driven model could deliver a 'best-fit curve', that is so clearly non-representative of its dataset, but still nonetheless managed to return a high score for RSqr (0.86). The lower data ranges are also of interest, since the each neurocomputing model adopts a very different curve in this region; highlighting a general lack of robustness which is almost certainly associated with the unusual data distributions identified in Section 2.2. PFT and PFA are, in contrast, seen to be robust solutions throughout in the respect that they do not attempt to introduce complex and unnecessary nonlinearities into the modelled relationship between SSC and Q. This situation is consistent for Experiment 1 and 2. Each rating curve model also supports smaller lower magnitude predictions and larger higher magnitude predictions in a better manner than any of their data-driven counterparts. The power to predict a near zero input-output relationship and to perform unencumbered higher magnitude extrapolations, beyond the range of the training dataset, is noted.

\section{DISCUSSION}

The evaluation of a model is dependent on one's subjective and / or objective hydrological insights into the processes operating in a catchment (developed from background information about a specific catchment and a priori knowledge of the physical, hydrological processes) and the ways in which 
these processes are / are not represented in data sets of adequate quality and completeness for the modelling task. Consequently, whether a model is accepted or rejected must include an element of qualitative evaluation above and beyond the quantitative indicators provided by goodness-of-fit statistics. In this context, therefore, the protocol outlined here relates to the steps necessary to formulate and apply that hydrological insight. In the case of this study, the hydrological insight is thus informed through a comprehensive appraisal of the physiographic, hydrological and sedimentological context of the catchment being model, plus consideration of the limitations associated with the data at that site. This insight is then used as an additional piece of information against which a model is judged.

Hydrological modellers should understand that each half of a limited two-water-year dataset, comprising daily records of water and sediment, will not contain sufficient information to support the proper capture and testing of heteroscedasticity, skewed frequency distributions and complex relationships occurring during storm events in an upland river on the island of Puerto Rico. Thus, the result is a unique model of the data; not of the comprehensive hydrological processes operating in the catchment. The previously-published solutions should, therefore,be caveated: emphasising the fact that a single year's record is far too limited to make broad hydrological generalisations, and that the study is concerned more with the testing of an algorithm than the generation of new hydrological knowledge. Kisi (2005) ranked sediment-discharge models, according to their level of statistical fit on the test period dataset. His preferred single-input single-output solutions were ordered from best to worst as follows: NFT-1, NNL-1, PFT-1 and SLR-1. The scientific position, however, is far more complicated since different orderings are identified in our dual reporting of statistical metrics for the training period and testing period datasets - something that was not apparent in the original paper. Mixed findings, moreover, suggest that instances of under- or over-fitting might exist across the different modelling solutions. This problem is commensurate with the use of fixed stopping conditions i.e. published models, might not be optimal calibrations of particular structures. The revised situation observed after the training and testing datasets had been swapped around, also confirms that the reported past and present numerical assessment of different methods is strongly biased by: (i) the unique nature of higher magnitude challenges involved in modelling small tropical island catchments; (ii) the small size and limited period dataset that was selected for model building and testing purposes and (iii) the partitioning process, that needed to provide equally balanced splitsample representations. Indeed, numerical inconsistencies across the board, mean that the selection of a superior modelling method under such conditions is, at best, unreliable: meaning that the original straightforward statistical assessment procedures can no longer be supported.

The use of regular data series plots, as required by our protocol, offers an alternative means of model selection based on two fundamental strategic considerations: (i) the relative importance of identified structures; and (ii) the operational demands of a proposed model. Figures 10 and 11 depict a modelled relationship that can be divided into upper, central and lower phases of fluvial sediment throughput. Each section of the plot corresponds to an integrated mix of identified hydrological processes and reported measurement uncertainties encapsulated in the downloaded dataset.

Modellers should understand that:

1. measurements obtained during major events, depicted in upper regions of the scatterplot, will be problematic. They will comprising a small number of extreme events, possessing strong hysteresis loops, that are difficult to assess with certainty using standard methods of instrumentation and/or daily reporting. Hence the need for on-site extrapolation by means of rating curves and, unfortunately, the related potential for introduced error. Thus quality, paucity and diversity of upper end recordings produces a region that is far less trustworthy and, all other things being equal, cannot be relied upon to deliver a sound assessment. The upper section, moreover, is expected to contain a broad scatter of points since a degree of uniqueness is an intrinsic component of extreme events on this island. Moreover, daily measurement records for upper magnitude events, are unlikely to provide a very accurate account of hydrological responses 
in the river. The result is that a small handful of isolated points, possessing high uncertainties, can easily impart an erroneous and disproportionate influence on either calibration and/or testing procedures, and goodness-of-fit statistics. Hence model differentiation on the basis of high flow events is not recommended.

2. Similarly, in the quiescent periods, depicted in lower regions of the scatterplot, one might also expect a strong degree of scatter since: (i) minor levels of fine-grained wash load, that can in most cases be attributed to an overland flow source, tend not to be strongly correlated with discharge; and (ii) accurate and representative recording of low-magnitude small-scale processes presents a number of technical and/or mechanical issues, such that instrumentation difficulties lead to the production of spurious scatter related to the aforementioned 'infilling' and/or 'binning' operations. Thus lower magnitude predictions are equally questionable. NF and NN data-driven predictions are elevated by a handful of larger events, such that the plot fails to intersect the origin. The provision of a 'floor' could in some instances be considered realistic. However, since most of the lower values for this catchment are found below the line plotted for our models, another outlier related issue appears to have arisen. The two power function models in contrast provide stable and consistent relationships for both experimental scenarios, across all regions of the solution space, and attempt to intersect the origin. Neither solution appears to be unduly influenced by upper and lower end outliers. No ceilings or floors exist so, in contrast to their data-driven counterparts, extrapolation can be performed in a sound and sensible manner.. Hence model differentiation on the basis of low flow events is not recommended.

The central region of each plot is as expected more consistent; displaying an imperfect, but nevertheless distinct, traditional log-log linear regression relationship. The majority of solutions under test adopted similar trajectories in this section and there is very little separation upon which to make a logical decision about a preferred individual model. However, in the upper and lower regions, model trajectories are quite different with $\mathrm{NF}$ and $\mathrm{NN}$ data-driven models possessing greater local nonlinearity which results in a model form that is difficult to rationalise. This leads to an important question: are flexible non-linear models, that deviate locally upwards and downwards from strong global trends in response to uncertain data records, operationally acceptable to hydrologists? The majority of NN solutions are more stable and consistent throughout and so might perhaps be considered as providing a more hydrologically sound model, since their curves do not display the higher-end susceptibilities of NF modelling. The observed upper end flattening out or ceiling effect nevertheless implies that a seasonally-driven supply limited process is occurring; something that can reasonably be postulated, but lacks clear supporting evidence in the dataset. NNL-2 is an exception: a larger internal architecture is required, but, regrettably, such activities exceeded our initial brief.

In accordance with our four-point data-driven appraisal modelling protocol, the above results must be contextualised, if a preferred solution is to be properly selected. It is clear that, when hydrological insight is applied, only the central region of each model represents a valid comparator. It is, therefore, difficult to select a preferred solution as most sensible solutions are very similar and cannot be easily distinguished in this range.

\section{CONCLUSIONS}

This single-input single-output re-analysis of Kisi's (2005) study in the context of DAMP and the decision sequences underpinning it, leads one to a very different conclusion from the original work. Given a poor initial dataset, a poor split of that dataset into poorer sub-sets, plus numerous measurement and recording uncertainties for the upper and lower magnitudes, log-log sediment rating curve methods provide a robust method, that fits the hydrological context, irrespective of overall statistical fit.

The application of our protocol here has made explicit the nature of opaque NF and NN modelling applications. NN and NF solutions are both prone to overfitting, requiring appropriate remedies, such as those presented in Giustolisi and Laucelli (2005). The reversal of modelling datasets, in pursuit of a consistent response, is one simple method that can be used to support or reject the assumption of 
equal representativeness amongst different sub-sets. The need to revisit previous published explorations and past modelled datasets to see if similar issues occur is important: it would, for example, be interesting to compare and contrast suspended sediment concentration outputs related to the reported application in this catchment of Fuzzy Differential Evolution (Kisi, 2009), Neural Differential Evolution (Kisi, 2010) and Linear Genetic Programming (Kisi and Guven, 2010).

It is clear from our analysis that hydrological context and knowledge of the catchment, are an essential component in an evaluation of the form of a hydrological model; and that the generation and use of regular series to elucidate model form should be standard practice in any sediment-discharge DDM. Fitting and evaluation of such models should, therefore, always involve more than a simple matter of calculating global statistics. Such numbers will sometimes camouflage, or otherwise overshadow, important issues and might suggest the inappropriate acceptance of a model that fails to adequately reflect hydrological context and data-quality issues or delivers output patterns that possess little or no scientific rationality. The nature of the solution is not irrelevant and obtaining a realistic model form is perhaps more important, in certain cases, than chasing superior global statistics implying that more effort should be devoted to examining such issues in reported applications.

There is a quotation about the strong persuasive power of numbers, which was popularised over 100 years ago by Mark Twain (1835-1910) but is still valid and pertinent today: "There are three kinds of lies: lies, damned lies, and statistics". It counsels that goodness-of-fit statistics associated with modelled hydrological data must not be evaluated or interpreted in isolation. From our simple singleinput single-output sediment-discharge case study it is evident that physical appreciation and geographical setting cannot be divorced from the application of data-driven modelling technology.

\section{REFERENCES}

Abrahart, R.J., Ab Ghani, N., Swan, J., 2009. DISCUSSION of 'An explicit neural network formulation for evapotranspiration'. Hydrological Sciences Journal 54, 382-388.

Abrahart, R.J., See, L.M. 2007., Neural network modelling of non-linear hydrological relationships. Hydrology and Earth System Sciences 11, 1563-1579.

Abrahart, R.J., See, L.M., Dawson, C.W., Shamseldin, A.Y., Wilby, R.L., 2010. Nearly Two Decades of Neural Network Hydrologic Modeling. In: Sivakumar, B. and Berndtsson, R. (eds) Advances in Data-Based Approaches for Hydrologic Modeling and Forecasting. Hackensack, NJ: World Scientific Publishing. pp. $267-346$.

Abrahart, R.J., See, L.M., Heppenstall, A.J., White, S.M., 2008. Neural Network Estimation of Suspended Sediment: Potential Pitfalls and Future Directions. In: Abrahart, R.J., See, L.M., Solomatine, D.P. (eds.) Practical Hydroinformatics: Computational Intelligence and Technological Developments in Water Applications. Springer-Verlag, Berlin and Heidelberg, pp. 139-161.

Alp, M., Ciğizoğlu, H.K., 2007. Suspended sediment load simulation by two artificial neural network methods using hydrometeorological data. Environmental Modelling \& Software 22, 2-13.

Aytek, A., Guven, A., Yuce, M.I., Aksoy, H., 2008. An explicit neural network formulation for evapotranspiration. Hydrological Sciences Journal 53, 893-904.

Aytek, A., Kisi, Ö., 2008. A genetic programming approach to suspended sediment modelling. Journal of Hydrology 351, 288-298.

Babovic, V., 2005. Data mining in hydrology. Hydrological Processes 19, 1511-1515.

Beven, K., 2006. A manifesto for the equifinality thesis. Journal of Hydrology 320, 18-36.

Buytaert, W., Reusser, D., Krause, S., Renaud, J-P., 2008. Why can't we do better than Topmodel? Hydrological Processes 22, 4175-4179.

Campbell, F.B., Bauder, H.A., 1940. A rating-curve method for determining silt-discharge of streams. Transactions of the American Geophysical Union 21, 603-607.

Ciğizoğlu, H.K., 2004. Estimation and forecasting of daily suspended sediment data by multi-layer perceptrons. Advances in Water Resources 27, 185-195. 
Ciğizoğlu, H.K., Alp, M., 2006. Generalized regression neural network in modelling river sediment yield. Advances in Engineering Software 37, 63-68.

Çimen, M., 2008. Estimation of daily suspended sediments using support vector machines. Hydrological Sciences Journal 53, 656-666.

Dawson, C.W., Abrahart, R.J., See, L.M., 2007. HydroTest: a web-based toolbox of evaluation metrics for the standardised assessment of hydrological forecasts. Environmental Modelling \& Software 22, 1034-1052.

Dawson, C.W., Abrahart, R.J., See, L.M., 2010. HydroTest: further development of a web resource for the standardised assessment of hydrological models. Environmental Modelling \& Software $25,1481-1482$.

Dawson, C.W. Abrahart, R.J. Shamseldin, A.Y., Wilby, R.L., 2006. Flood estimation at ungauged sites using artificial neural networks. Journal of Hydrology 319, 391-409

Dawson, C.W. Harpham, C. Wilby, R.L., Chen, Y., 2002. An Evaluation of Artificial Neural Network Techniques for Flow Forecasting in the River Yangtze, China. Hydrology and Earth System Sciences 6, 619-626.

Diaz-Ramirez, J.N., Perez-Alegria, L.R., McAnally, W.H., 2008. Hydrology and Sediment Modeling Using HSPF/BASINS in a Tropical Island Watershed. Transactions of the ASABE 51, 15551565.

Dunne, T., 1979. Sediment yield and land use in tropical catchments. Journal of Hydrology 42, 281300.

Ferguson, R.I., 1986. River Loads Underestimated by Rating Curves. Water Resources Research 22, 74-76.

Glysson, G.D., 1987. Sediment-Transport Curves. US Geological Survey Open File Report 87-218. pp.47.

Giustolisi, O., Laucelli, D., 2005. Improving generalization of artificial neural networks in rainfallrunoff modelling. Hydrological Sciences Journal 50, 439-457.

Helmer, E.H., Ramos, O., Lopez, T., Del, M., Quinones, M., Diaz, W., 2002. Mapping the forest type and land cover of Puerto Rico, a component of the Caribbean biodiversity hotspot. Caribbean Journal of Science 38, 165-183.

Ho, F.P., Scwerde, R.W., Goodyear, H.V., 1975. Some Climatological Characteristics of Hurricanes and Tropical Storms, Gulf and East Coast of the United States. NOAA Technical Report NWS15 (COM-7511-88).

Jain, S.K., 2001. Development of Integrated Sediment Rating Curves Using ANNs. ASCE Journal of Hydraulic Engineering 127, 30-37.

Jain, S.K., 2008. Development of Integrated Discharge and Sediment Rating Relation Using a Compound Neural Network. ASCE Journal of Hydrologic Engineering 13, 124-131.

Jang, J-S.R., 1993. ANFIS: Adaptive-Network-based Fuzzy Inference System. IEEE Transactions on Systems, Man, and Cybernetics 23, 665-685.

Jang, J-S.R., Sun, C-T., Mizutani, E., 1997. Neuro-Fuzzy and Soft Computing: a computational approach to learning and machine intelligence, Prentice Hall, Upper Saddle River, NJ, USA.

Kişi, Ö., 2004a. Multi-layer perceptrons with Levenberg-Marquardt training algorithm for suspended sediment concentration prediction and estimation. Hydrological Sciences Journal 49, 10251040.

Kisi, Ö., 2004b. Daily suspended sediment modelling using a fuzzy differential evolution approach. Hydrological Sciences Journal 49, 183-197.

Kisi, Ö., 2005. Suspended sediment estimation using neuro-fuzzy and neural network approaches. Hydrological Sciences Journal 50, 683-696.

Kisi, Ö., 2009. Evolutionary fuzzy models for river suspended sediment concentration estimation. Journal of Hydrology 372, 68-79.

Kişi, Ö., 2010. River suspended sediment concentration modeling using a neural differential evolution approach. Journal of Hydrology 389, 227-235.

Kisi, Ö., Guven, A., 2010. A machine code-based genetic programming for suspended sediment concentration estimation. Advances in Engineering Software 41, 939-945. 
Kisi, Ö., Haktanir, T., Ardiclioglu, M., Ozturk, O., Yalcin, E., Uludag, S., 2009. Adaptive neuro-fuzzy computing technique for suspended sediment estimation. Advances in Engineering Software 40, 438-444.

Kisi, O., Karahan, M.E., Sen, Z., 2006. River suspended sediment modeling using fuzzy logic approach. Hydrological Processes 20, 4351-4362.

Kisi, Ö., Yuksel, I., Dogan, E., 2008. Modelling daily suspended sediment of rivers in Turkey using several data-driven techniques. Hydrological Sciences Journal 53, 1270-1285.

Larsen, M.C., 1997. Tropical geomorphology and geomorphic work: A study of geomorphic processes and sediment and water budgets in montane humid-tropical forested and developed watersheds, Puerto Rico. Unpublished Ph.D. Thesis, Geography Department, University of Colorado, Boulder, Colorado, USA. 341 pp.

Larsen, M.C., Torres-Sanchez, A.J., 1998. The frequency and distribution of recent landslides in three montane tropical regions of Puerto Rico. Geomorphology 24, 309-331.

Lee, H-Y., Lin, Y-T., Chiu, Y-J., 2006. Quantitative Estimation of Reservoir Sedimentation from Three Typhoon Events. ASCE Journal of Hydrologic Engineering 11, 362-370.

Minns A.W., Hall M.J., 1996. Artificial neural networks as rainfall-runoff models. Hydrological Sciences Journal 41, 399-417.

Mount, N.J., Abrahart, R.J., in press. Load or concentration, logged or unlogged? Addressing ten years of uncertainty in neural network suspended sediment prediction. To appear in: Hydrological Processes [HYP-10-0638.R1].

Neuman, C.J., Jarvinen, B.R., Pike, A.C., 1990. Tropical cyclones of the North Atlantic Ocean, 19711986 (with storm tracks through 1989). National Climatic Data Center Historical Climatology Series 6. 186. pp.

Oreskes, N., 2003. The Role of Quantitative Models in Science, in: Canham, C.D.W., Cole,J., Lauenroth, W.K. (Eds.), Models in Ecosystem Science, Princeton University Press, Princeton, NJ, USA, pp. 13-31.

Parés-Ramos, I.K., Gould, W.A., Aide, T.M., 2008. Agricultural abandonment, suburban growth, and forest expansion in Puerto Rico between 1991 and 2000. Ecology and Society 13, 1. [online]

Partal, T., Cigizoglu, H.K., 2008. Estimation and forecasting of daily suspended sediment data using wavelet-neural networks. Journal of Hydrology 358, 317-331.

Priddy, K.L., Keller, P.E., 2005. Artificial neural networks: An Introduction, SPIE-International Society for Optical Engineering Press, Bellingham, WA, USA.

Rajaee, T., Mirbagheri, S.A., Nourani, V., Alikhani, A., 2010. Prediction of daily suspended sediment load using wavelet and neuro-fuzzy combined model. International Journal of Environmental Science and Technology 7, 93-110.

Sayed, T., Tavakolie, A., Razavi, A., 2003. Comparison of adaptive network based fuzzy inference systems and B-spline neuro-fuzzy mode choice models. Journal of Computing in Civil Engineering 17, 123-130.

Scatena, F.N., Larsen, M.C., 1991. Physical aspects of Hurricane Hugo in Puerto Rico. Biotropica 23, 317-323.

Şen, Z., Altunkaynak, A., 2003. Fuzzy awakening in rainfall-runoff modeling. Nordic Hydrology 35, 31-43.

Solomatine, D.P., See, L.M, Abrahart, R.J., 2008. Data-driven modelling: concepts, approaches and experiences, in: Abrahart, R.J., See, L.M., Solomatine, D.P. (Eds.), Practical Hydroinformatics: Computational Intelligence and Technological Developments in Water Application, SpringerVerlag, Berlin and Heidelberg, Germany, pp. 17-30.

Todini, E., 2007. Hydrological catchment modelling: past, present and future. Hydrology and Earth System Sciences 11, 468-482

USGS, 2000. Luquillo Mountains, Peurto Rico - A Water, Energy and Biogeochemical Budgets Program Site. USGS Fact Sheet 163-99.

Warne, A.G., Webb, R.M.T., Larsen, M.C., 2005. Water, Sediment, and Nutrient Discharge Characteristics of Rivers in Puerto Rico, and their Potential Influence on Coral Reefs. USGS Scientific Investigations Report 2005-5206. 186 pp.

Williams, G.P., 1989. Sediment concentration versus water discharge during single hydrologic events in rivers. Journal of Hydrology 111, 89-106. 
Table 1: USGS site description for monitoring station no. 50056400

\begin{tabular}{|l|l|}
\hline Administrative Unit: & Juncos Municipio, Puerto Rico \\
\hline Hydrologic Unit: & 21010005 \\
\hline Latitude: & $18^{\circ} 12^{\prime} 58^{\prime \prime}$ (NAD27) \\
\hline Longitude: & $65^{\circ} 55^{\prime} 34 "$ (NAD27) \\
\hline Elevation: & $97.5 \mathrm{~m}$ asl (NGVD29) \\
\hline Drainage Area: & $42.5 \mathrm{~km}^{2}$ \\
\hline
\end{tabular}

Table 2: Statistical description of split sample datasets

\begin{tabular}{|l|r|r|r|r|}
\hline & \multicolumn{2}{|c|}{$\mathrm{Q}\left(\mathrm{m}^{3} \mathrm{~s}^{-1}\right)$} & \multicolumn{2}{c|}{$\mathrm{S}\left(\mathrm{mg} \mathrm{l}^{-1}\right)$} \\
\hline & Set A & \multicolumn{1}{c|}{ Set B } & Set A & \multicolumn{1}{c|}{ Set B } \\
\hline Water Year: & 1994 & 1995 & 1994 & 1995 \\
\hline Minimum: & 0.04 & 0.05 & 2.00 & 4.00 \\
\hline Mean: & 0.61 & 1.05 & 41.63 & 71.47 \\
\hline Maximum: & 35.10 & 24.60 & 1200.00 & 1090.00 \\
\hline Range: & 35.06 & 24.55 & 1198.00 & 1086.00 \\
\hline Std. Dev.: & 2.06 & 2.47 & 105.94 & 147.40 \\
\hline Skewness: & 13.59 & 5.73 & 7.48 & 4.32 \\
\hline Kurtosis: & 218.72 & 39.80 & 66.68 & 21.51 \\
\hline Count: & 364 & 364 & 364 & 364 \\
\hline
\end{tabular}

$\mathrm{Q}=$ discharge; $\mathrm{S}=$ suspended sediment concentration 
Table 4: NF parameter settings and rule set for Equations 1-8

Number of MFs -2
NFT-1
Type of MF - triangular
Triangular parameters:
\begin{tabular}{|l|l|l|l|}
\hline & a & b & c \\
\hline $\mathbf{M F}_{1}$ & -35.02 & 0.0714 & 35.03 \\
\hline $\mathbf{M F}_{2}$ & 0.0070 & 35.13 & 70.16 \\
\hline
\end{tabular}

Number of MFs -2

Type of MF - triangular

Triangular parameters:

\begin{tabular}{|l|l|l|l|}
\hline & a & b & c \\
\hline $\mathbf{M F}_{\mathbf{1}}$ & -24.5 & 0.0135 & 24.64 \\
\hline $\mathbf{M F}_{\mathbf{2}}$ & 0.1177 & 24.56 & 49.15 \\
\hline
\end{tabular}

Rules:

If $\mathrm{Q}$ is $\mathrm{MF}_{1}$ then $\mathrm{S}_{1}$ is $-226.4 \mathrm{Q}+4.912$

Rules:

If $\mathrm{Q}$ is $\mathrm{MF}_{1}$ then $\mathrm{S}_{1}$ is $29.79 \mathrm{Q}+3.583$

If $\mathrm{Q}$ is $\mathrm{MF}_{2}$ then $\mathrm{S}_{2}$ is $-270.7 \mathrm{Q}+10560$

If $\mathrm{Q}$ is $\mathrm{MF}_{2}$ then $\mathrm{S}_{2}$ is $-11.49 \mathrm{Q}+1274$

Number of MFs -2

\section{NFG-1}

Type of MF - Gaussian

Number of MFs - 2

\section{NFG-2}

Gaussian parameters:

\begin{tabular}{|l|l|l|}
\hline & $\boldsymbol{\sigma}$ & $\boldsymbol{c}$ \\
\hline $\mathbf{M F}_{\mathbf{1}}$ & 14.93 & 0.0324 \\
\hline $\mathbf{M F}_{\mathbf{2}}$ & 14.95 & 35.05 \\
\hline
\end{tabular}

Type of MF - Gaussian

Gaussian parameters:

\begin{tabular}{|l|l|l|}
\hline & $\boldsymbol{\sigma}$ & $\boldsymbol{c}$ \\
\hline $\mathbf{M F}_{\mathbf{1}}$ & 10.47 & 0.0280 \\
\hline $\mathbf{M F}_{\mathbf{2}}$ & 10.48 & 24.54 \\
\hline
\end{tabular}

Rules:

If $Q$ is $\mathrm{MF}_{1}$ then $\mathrm{S}_{1}$ is $37.51 Q-169.9$

Rules:

If $Q$ is $\mathrm{MF}_{1}$ then $\mathrm{S}_{1}$ is $100.6 Q+43.56$

If $Q$ is $\mathrm{MF}_{2}$ then $\mathrm{S}_{2}$ is $-49.34 Q+2775$

If $Q$ is $\mathrm{MF}_{2}$ then $\mathrm{S}_{2}$ is $73.11 Q-776.3$ 
Table 5: NN parameter settings for Equations 9-13

\begin{tabular}{lrrrr}
\hline & \multicolumn{1}{c}{ NNL-1 } & \multicolumn{1}{c}{ NNN-1 } & \multicolumn{1}{c}{ NNL-2 } & \multicolumn{1}{c}{ NNN-2 } \\
W1 (weight of hidden layer) & 11.4319 & -6.1670 & 7.8000 & -5.0555 \\
W2 (weight of output layer) & 0.8359 & -10.5612 & 0.6846 & -23.1867 \\
B1 (bias factor of hidden layer) & -2.6970 & 0.0985 & -2.0471 & -1.3031 \\
B2 (bias factor of output layer) & -0.0410 & 1.3425 & -0.0721 & 1.1041 \\
$\mathrm{Q}_{\min }$ (minimum observed Q) & 0.0396 & 0.0396 & 0.0510 & 0.0510 \\
$\mathrm{Q}_{\max }$ (maximum observed Q) & 35.1000 & 35.1000 & 24.6000 & 24.6000 \\
$\mathrm{~S}_{\min }$ (minimum observed S) & 2.0000 & 2.0000 & 4.0000 & 4.0000 \\
$\mathrm{~S}_{\max }$ (maximum observed S) & 1200.0000 & 1200.0000 & 1090.0000 & 1090.0000 \\
\hline
\end{tabular}


Table 6: Numerical verification against earlier paper

\begin{tabular}{lcccccc}
\hline & \multicolumn{2}{c}{ RMSE } & \multicolumn{2}{c}{ RSqr } & \multicolumn{2}{c}{ TE (\%) } \\
\hline Model & Kisi (2005) & Abrahart et al. & Kisi (2005) & Abrahart et al. & Kisi (2005)* & Abrahart et al. \\
\hline NFT-1 & 51.89 & 51.96 & 0.88 & 0.88 & -1.82 & -1.54 \\
NNL-1 & 54.57 & 57.10 & 0.87 & 0.85 & -1.99 & 2.93 \\
PFT-1 & 58.38 & 58.30 & 0.85 & 0.85 & -7.36 & -7.58 \\
SLR-1 & 74.41 & 74.12 & 0.84 & 0.84 & -29.24 & -29.07 \\
\hline
\end{tabular}

* recalculated, using reported 'total estimated sediment' 
Table 7: Evaluation statistics for Experiment 1

\begin{tabular}{crrrrrrr}
\hline & \multicolumn{3}{c}{ Model Under Test } & & \multicolumn{2}{c}{ Traditional Benchmarking } \\
\hline $\begin{array}{c}\text { SET A } \\
\text { (Training) }\end{array}$ & NFT-1 & NFG-1 & NNL-1 & NNN-1 & PFT-1 & PFA-1 & SLR-1 \\
\hline MAE & 13.04 & 12.86 & $\mathbf{1 1 . 1 4}$ & 12.26 & 13.08 & 14.27 & 21.23 \\
ME & $\mathbf{0 . 0 0}$ & $\mathbf{0 . 0 0}$ & 3.37 & 1.01 & 5.20 & 0.27 & $\mathbf{0 . 0 0}$ \\
RMSE & 65.36 & 65.07 & 65.16 & $\mathbf{6 4 . 9 6}$ & 74.83 & 80.16 & 72.29 \\
MARE & 0.52 & 0.74 & 0.59 & 0.79 & $\mathbf{0 . 2 8}$ & 0.38 & 1.44 \\
MRE & -0.41 & -0.64 & -0.46 & -0.67 & $-\mathbf{0 . 0 9}$ & -0.23 & -1.34 \\
MSRE & 0.45 & 1.22 & 0.90 & 1.62 & $\mathbf{0 . 1 3}$ & 0.22 & 5.59 \\
RSqr & $\mathbf{0 . 6 2}$ & $\mathbf{0 . 6 2}$ & $\mathbf{0 . 6 2}$ & $\mathbf{0 . 6 2}$ & 0.55 & 0.55 & 0.53 \\
TE (\%) & $\mathbf{0 . 0 0}$ & $\mathbf{0 . 0 0}$ & -0.03 & -0.76 & 26.38 & 43.51 & $\mathbf{0 . 0 0}$ \\
\hline & & & & & & & \\
\hline SET B & $\mathbf{N F T - 1}$ & $\mathbf{N F G - 1}$ & $\mathbf{N N L - 1}$ & $\mathbf{N N N - 1}$ & $\mathbf{P F T - 1}$ & PFA-1 & SLR-1 \\
(Testing) & & & & & & & \\
\hline MAE: & $\mathbf{2 1 . 7 9}$ & 26.07 & 25.09 & 24.91 & 25.44 & 23.45 & 35.38 \\
ME & $\mathbf{0 . 4 8}$ & -1.69 & 4.57 & 3.17 & 10.65 & 2.43 & 13.26 \\
RMSE & $\mathbf{5 1 . 9 6}$ & 62.95 & 57.10 & 54.69 & 58.30 & 57.23 & $\mathbf{7 4 . 1 2}$ \\
MARE & 0.78 & 0.89 & 0.76 & 0.89 & $\mathbf{0 . 6 1}$ & 0.69 & 1.31 \\
MRE & -0.64 & -0.73 & -0.52 & -0.70 & $\mathbf{- 0 . 3 3}$ & -0.51 & -1.08 \\
MSRE & 1.83 & 1.96 & 1.37 & 1.86 & $\mathbf{1 . 0 6}$ & 1.49 & 3.63 \\
RSqr & $\mathbf{0 . 8 8}$ & 0.84 & 0.85 & 0.86 & 0.85 & 0.85 & 0.84 \\
TE (\%) & -1.54 & 14.51 & 2.93 & $-\mathbf{0 . 8 4}$ & -7.58 & 4.95 & -29.07 \\
\hline NB: TOp & & & & & & & \\
\hline
\end{tabular}

NB: Top score per metric in bold 
Table 8: Evaluation statistics for Experiment 2

\begin{tabular}{|c|c|c|c|c|c|c|c|}
\hline \multirow[b]{2}{*}{$\begin{array}{c}\text { SET B } \\
\text { (Training) }\end{array}$} & \multicolumn{4}{|c|}{ Model Under Test } & \multicolumn{3}{|c|}{ Traditional Benchmarking } \\
\hline & NFT-2 & NFG-2 & NNL-2 & NNN-2 & PFT-2 & PFA-2 & SLR-2 \\
\hline MAE & 20.12 & 19.64 & 55.08 & 22.08 & 26.31 & 27.65 & 29.03 \\
\hline ME & 0.00 & 0.00 & 51.93 & 0.30 & 3.09 & -8.06 & 0.00 \\
\hline RMSE & 51.22 & 50.32 & 81.47 & 52.72 & 73.31 & 92.16 & 59.42 \\
\hline MARE & 0.63 & 0.63 & 2.14 & 0.78 & 0.54 & 0.61 & 1.24 \\
\hline MRE & -0.47 & -0.31 & 2.09 & -0.62 & -0.19 & -0.38 & -1.10 \\
\hline MSRE & 1.55 & 1.60 & 7.35 & 1.71 & 0.91 & 1.33 & 3.39 \\
\hline RSqr & 0.88 & 0.88 & 0.86 & 0.87 & 0.83 & 0.83 & 0.84 \\
\hline TE (\%) & 0.00 & 0.00 & -32.80 & -2.85 & 22.75 & 42.84 & 0.00 \\
\hline $\begin{array}{c}\text { SET A } \\
\text { (Testing) }\end{array}$ & NFT-2 & NFG-2 & NNL-2 & NNN-2 & PFT-2 & PFA-2 & SLR-2 \\
\hline MAE & 12.84 & 15.59 & 43.96 & 13.20 & 13.32 & 17.10 & 19.84 \\
\hline ME & 1.41 & 0.07 & 43.96 & 0.21 & 2.91 & -3.41 & -5.70 \\
\hline RMSE & 66.25 & 76.98 & 81.14 & 65.95 & 103.21 & 121.53 & 80.66 \\
\hline MARE & 0.35 & 0.47 & 2.87 & 0.58 & 0.19 & 0.27 & 1.24 \\
\hline MRE & -0.20 & 0.17 & 2.87 & -0.47 & 0.08 & -0.07 & -1.18 \\
\hline MSRE & 0.20 & 0.41 & 15.47 & 0.69 & 0.09 & 0.13 & 3.70 \\
\hline RSqr & 0.61 & 0.59 & 0.62 & 0.61 & 0.52 & 0.52 & 0.53 \\
\hline TE (\%) & -7.27 & 42.88 & -37.24 & -4.63 & 78.96 & 108.24 & 41.44 \\
\hline
\end{tabular}

NB: Top score per metric in bold 


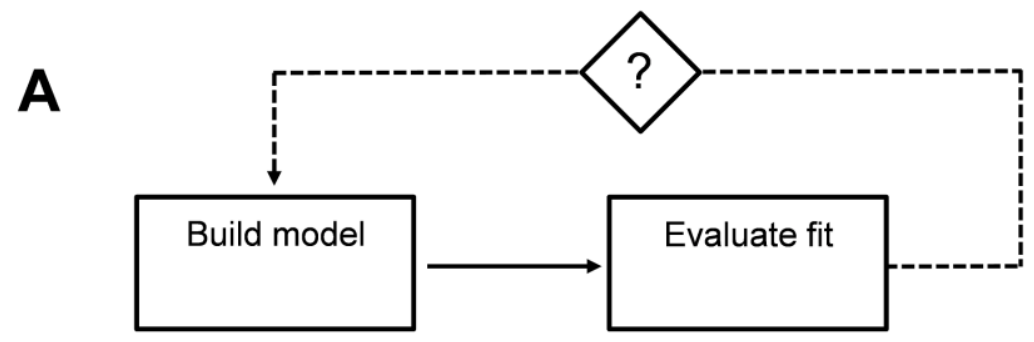

B

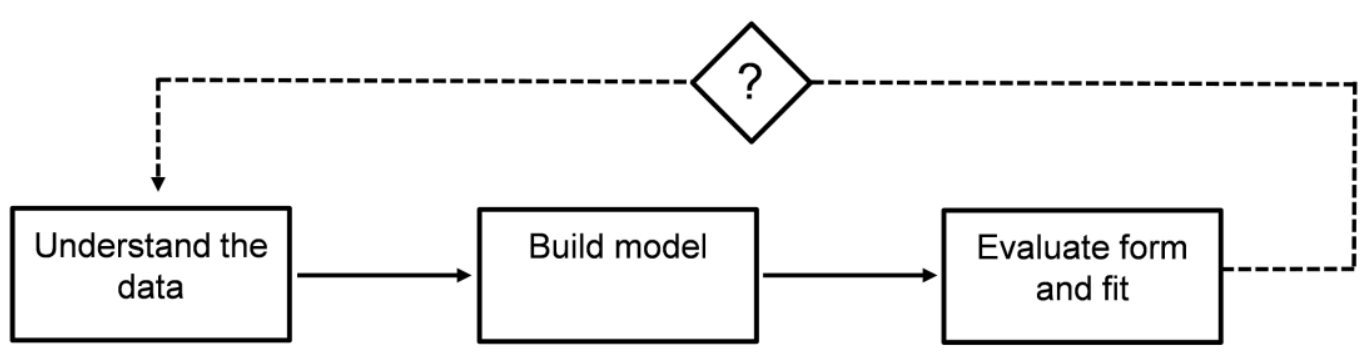

Figure 1: The decision sequences commonly used in the evaluation and selection of a data-driven (A) and conventional, empirical (B) rating curve model. 


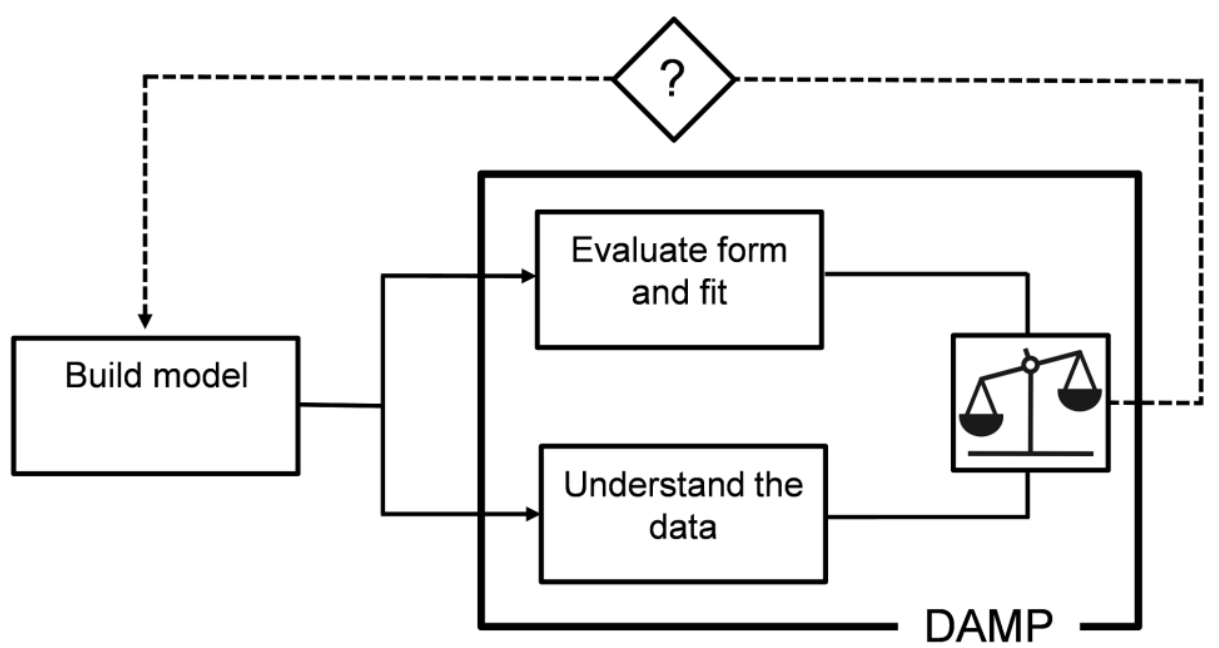

Figure 2: The decision sequence underpinning DAMP, in which model selection is made on the basis of a parallel evaluation of both 1) the form of the model and its fit to the data and 2) the hydrological and physiographic context which underpins the understanding of the dataset to be modelled. 
ATLANTIC OCEAN

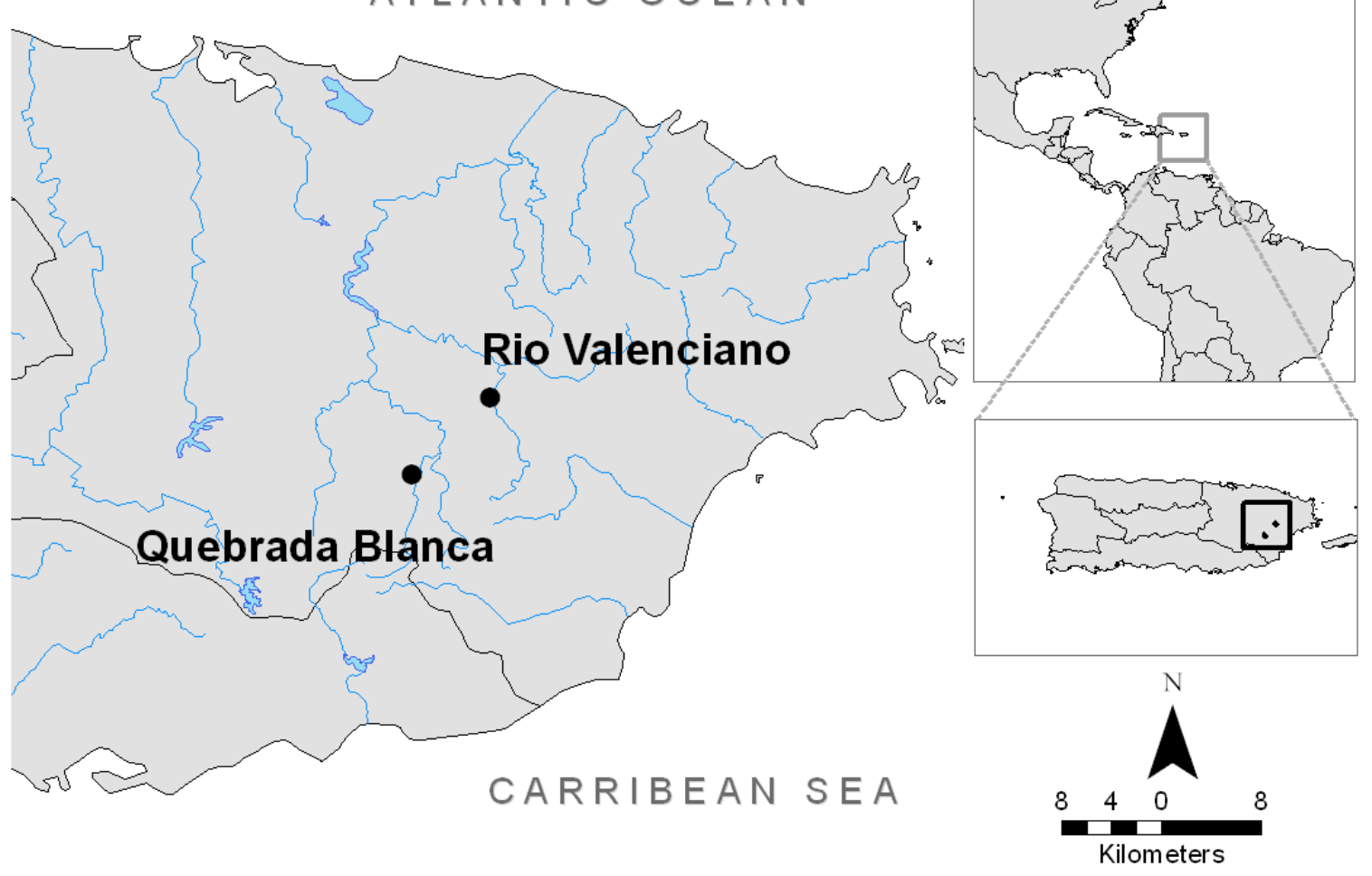

Figure 3: Location of two original gauging stations in Puerto Rico 

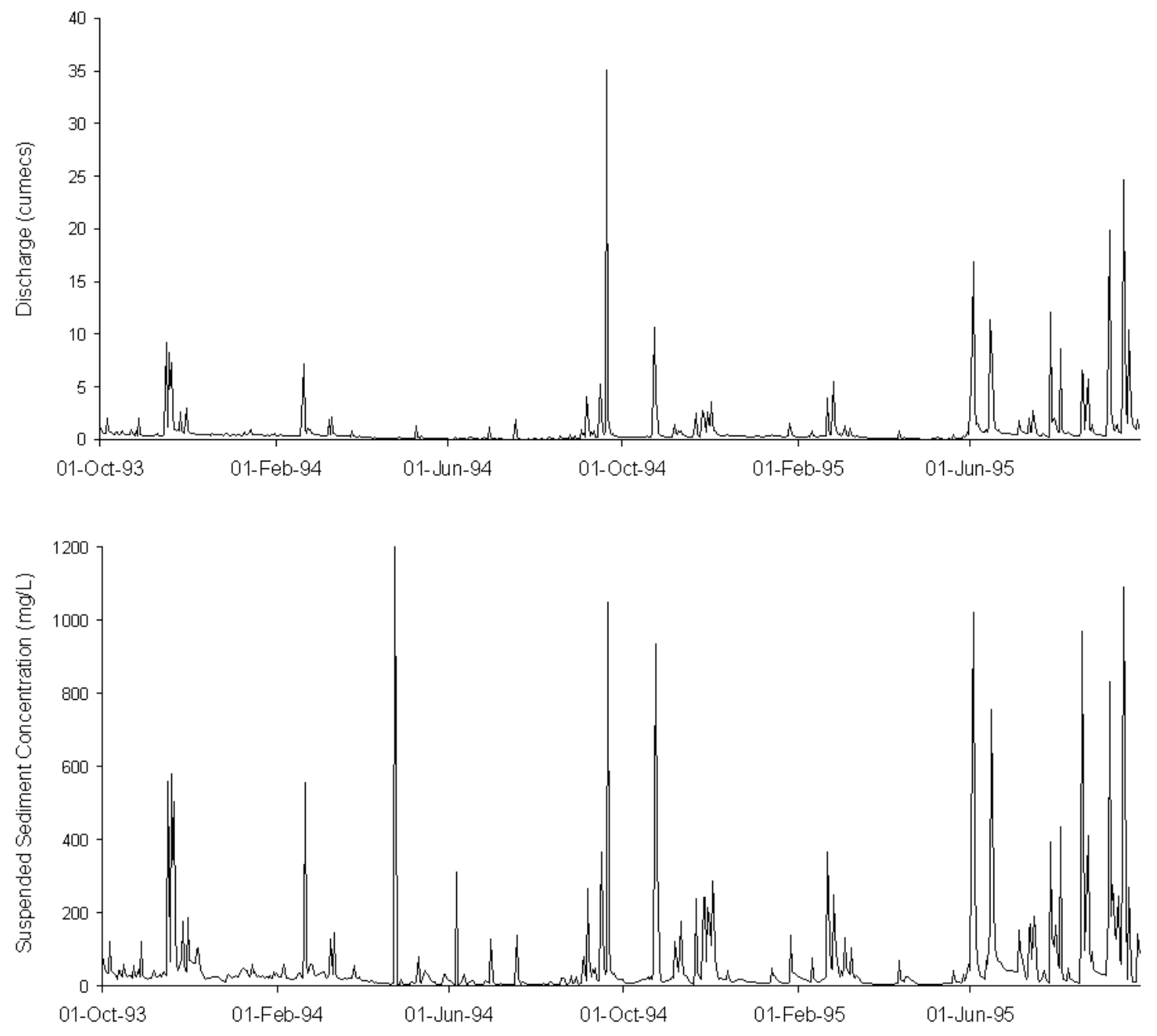

Figure 4: Time series plot showing seasonal pattern and peaked nature of downloaded dataset 


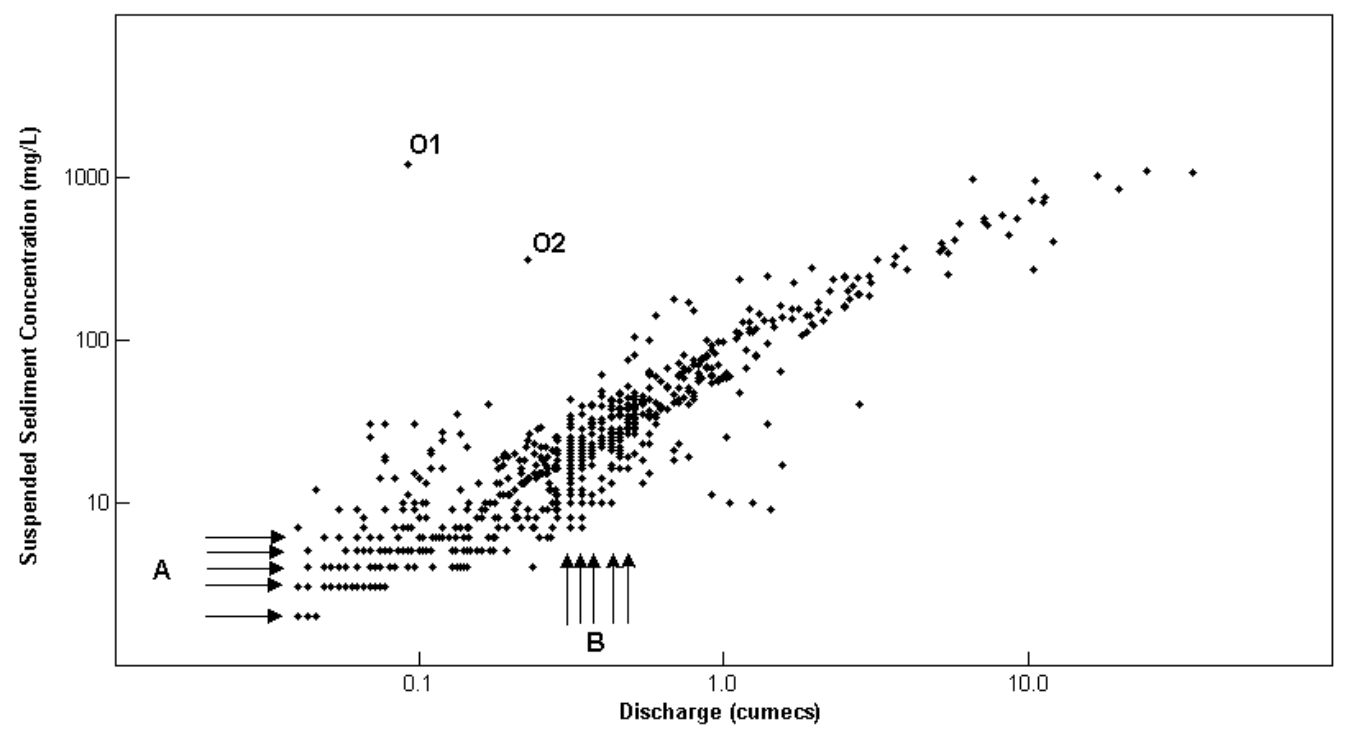

Figure 5: Log-log plot showing primary and secondary structures in downloaded dataset 


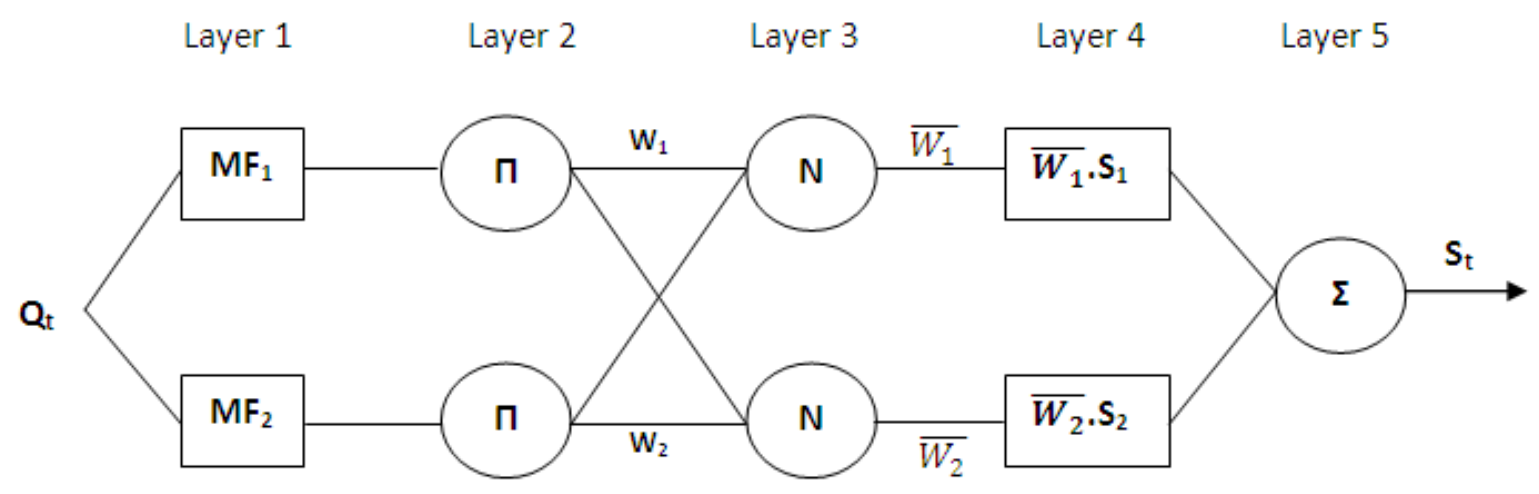

Figure 6: NF architecture for one-input two-membership-function model 


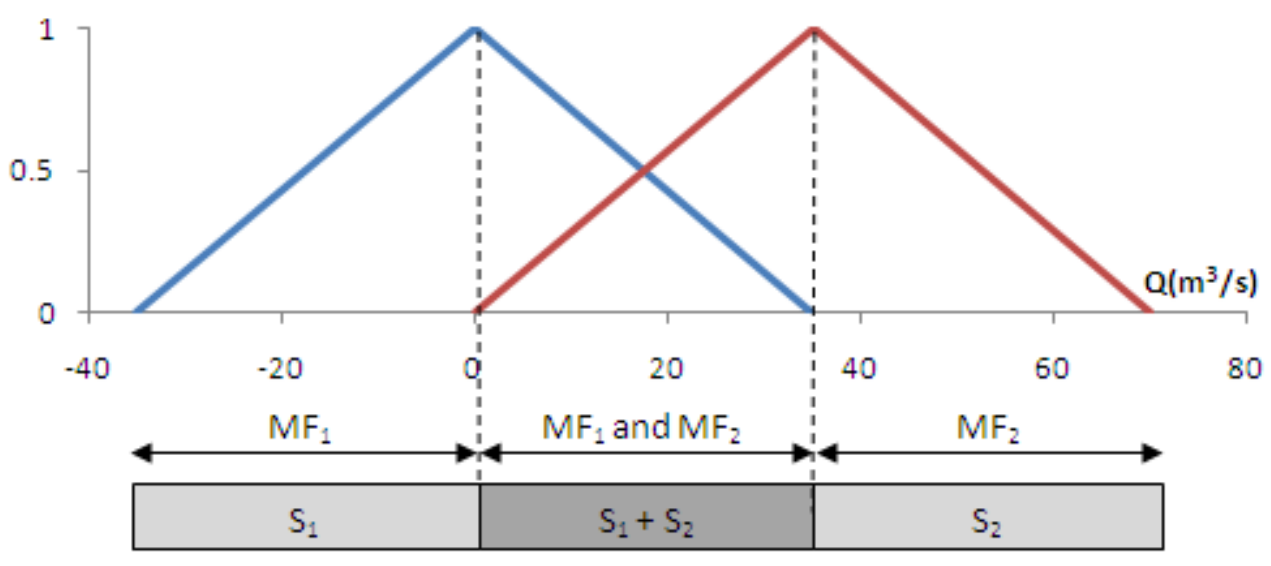

Figure 7: Triangular membership function: $\mathrm{MF}_{1}$ and $\mathrm{MF}_{2}$ for NFT-1 

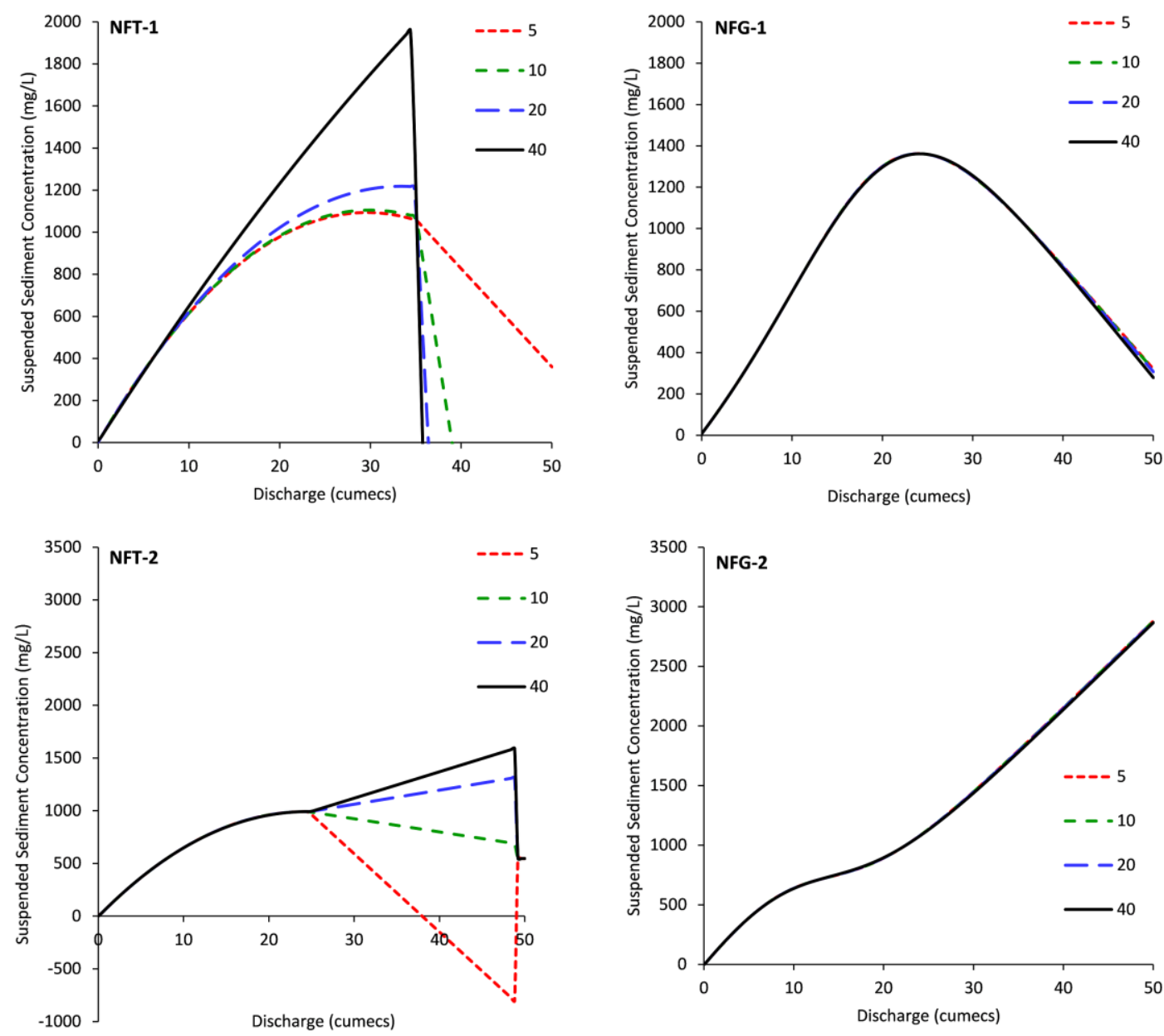

Figure 8: NFT and NFG trials for different number of iterations 


$$
\text { Input layer Hidden layer Output layer }
$$

(i) (j) (k)

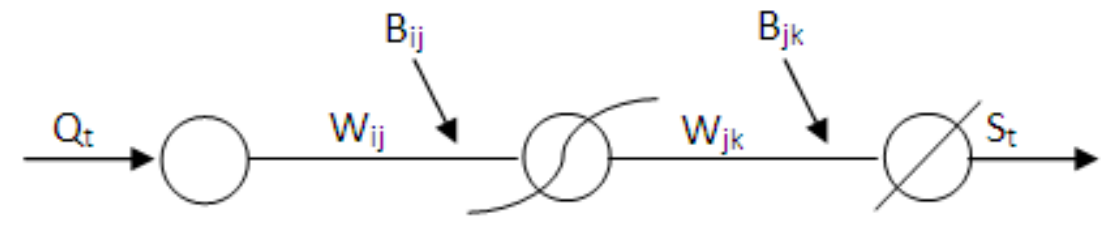

Figure 9: NN architecture for a 1:1:1 model, containing non-linear intermediate hidden layer and linear final output layer processing units 

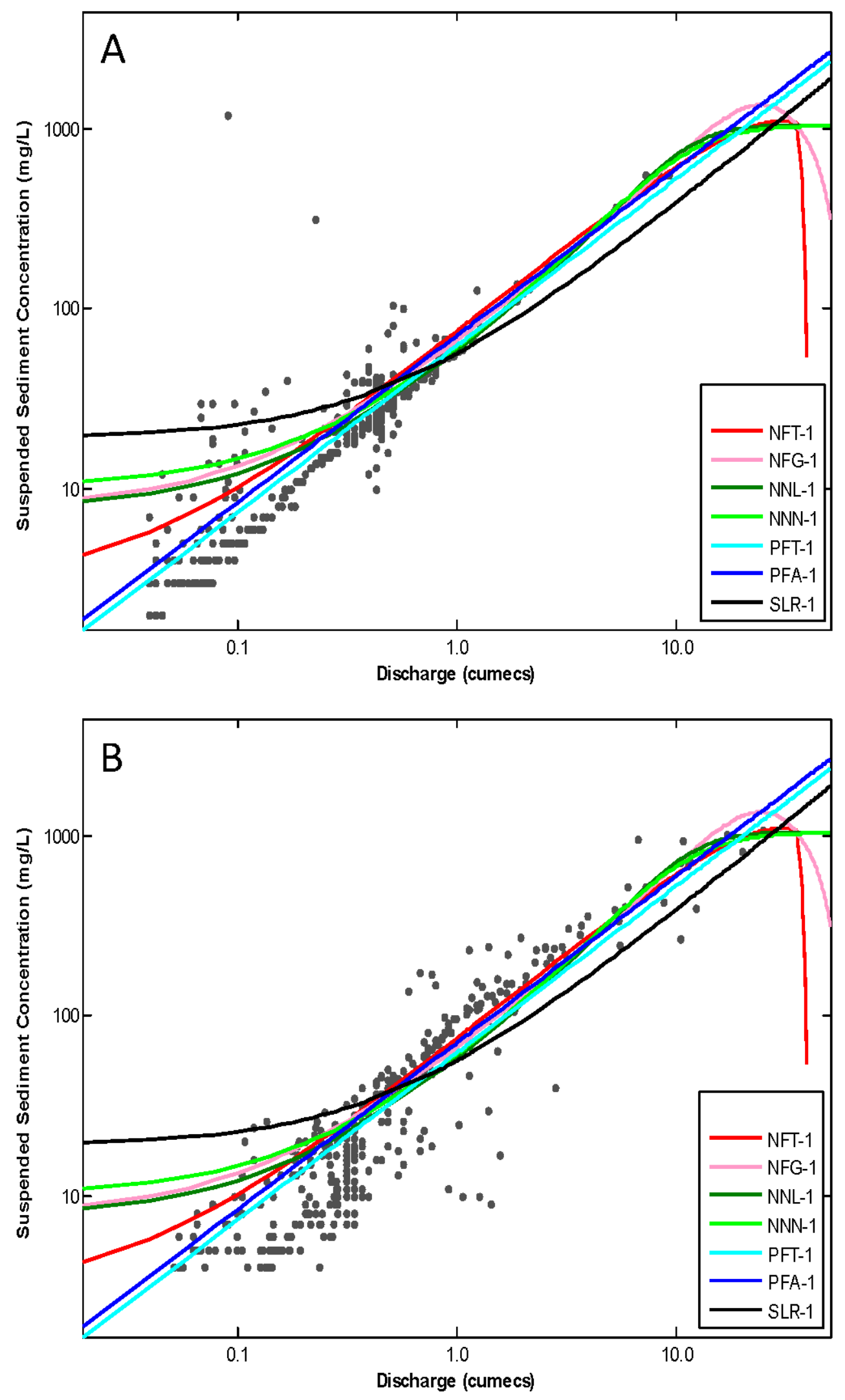

Figure 10: Models plotted against a) training dataset and b) testing dataset for Experiment 1 

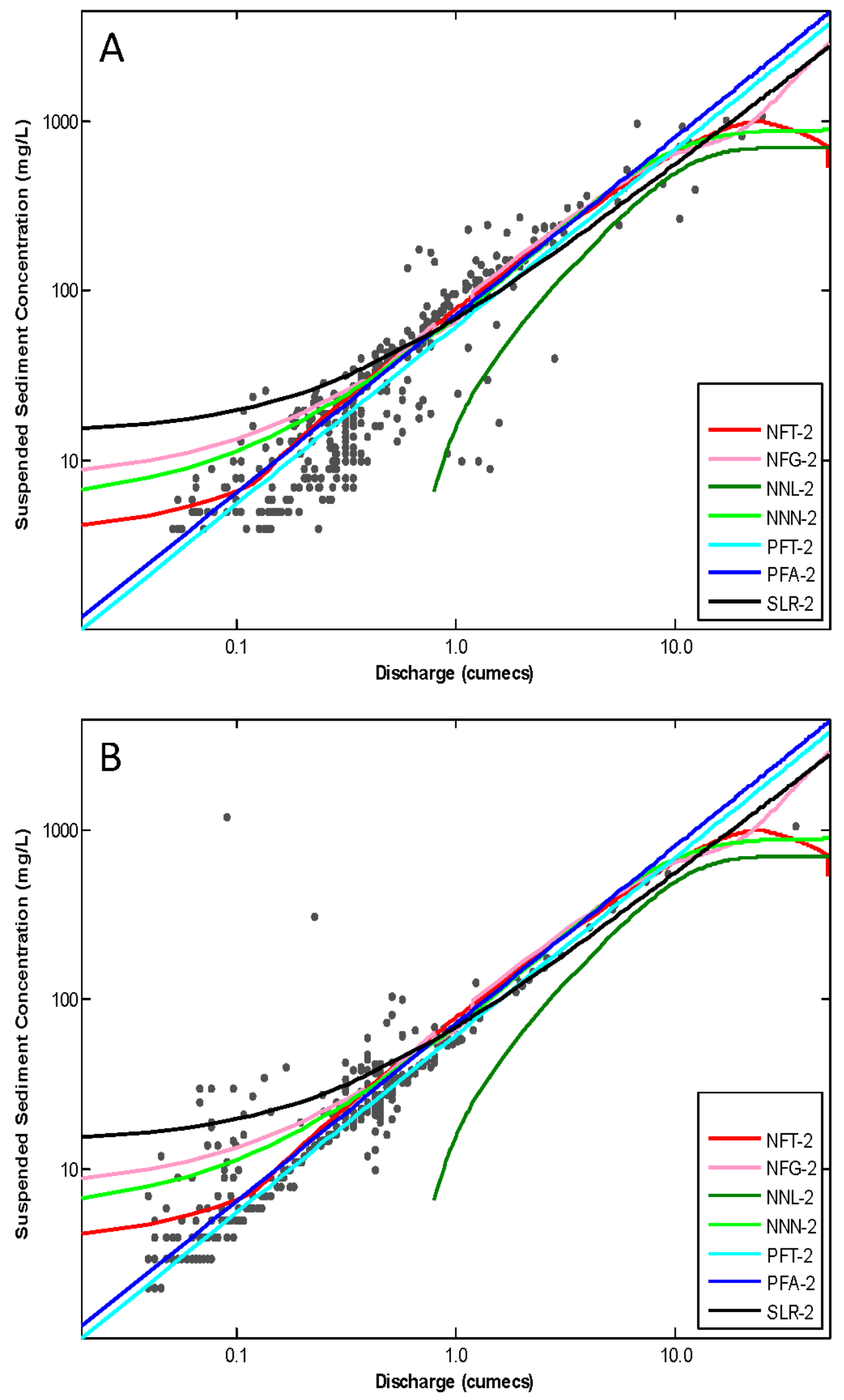

Figure 11: Models plotted against a) training dataset and b) testing dataset for Experiment 2 